\title{
Deceleration and trapping of ammonia using time-varying electric fields
}

\author{
Hendrick L. Bethlem, Floris M. H. Crompvoets, Rienk T. Jongma, Sebastiaan Y. T. van de Meerakker, and Gerard Meijer \\ FOM-Institute for Plasma Physics Rijnhuizen, P.O. Box 1207, NL-3430 BE Nieuwegein, The Netherlands \\ and Department of Molecular and Laser Physics, University of Nijmegen, Toernooiveld 1, NL-6525 ED Nijmegen, The Netherlands
}

(Received 1 February 2002; published 10 May 2002)

\begin{abstract}
A polar molecule experiences a force in an inhomogeneous electric field. Using this force, neutral molecules can be decelerated and trapped. It is shown here that this can in principle be done without loss in phase-space density. Using a series of 64 pulsed inhomogeneous electric fields a supersonic beam of ammonia molecules $\left({ }^{14} \mathrm{NH}_{3},{ }^{14} \mathrm{ND}_{3},{ }^{15} \mathrm{ND}_{3}\right)$ is decelerated. Subsequently, the decelerated molecules are loaded into an electrostatic quadrupole trap. Densities on the order of $10^{7}$ molecules $/ \mathrm{cm}^{3}$ at a temperature of $25 \mathrm{mK}$ are obtained for ${ }^{14} \mathrm{ND}_{3}$ and ${ }^{15} \mathrm{ND}_{3}$ separately and simultaneously. This corresponds to a phase-space density in the trap of $2 \times 10^{-13}, 50$ times less than the initial phase-space density in the beam.

DOI: 10.1103/PhysRevA.65.053416

PACS number(s): 33.80.Ps, 33.55.Be, 39.10.+j
\end{abstract}

\section{INTRODUCTION}

Currently there is great interest in the physics of cold molecules [1-5]. There are now three techniques that have been able to produce samples of trapped cold molecules. In the first technique, homonuclear diatomic molecules are formed by photoassociation of cold atoms [6,7]. In this way ultracold $\mathrm{H}_{2}$ [8], $\mathrm{He}_{2}^{*}$ [9], $\mathrm{Li}_{2}$ [10], $\mathrm{Na}_{2}$ [11], $\mathrm{K}_{2}$ [12,13], $\mathrm{Ca}_{2}$ [14], $\mathrm{Rb}_{2}[15,16]$, and $\mathrm{Cs}_{2}[17]$ have been formed. The translational temperature of the molecules can be as low as that of the atoms from which they are formed. Recently, photoassociation of atoms has been demonstrated in a Bose-Einstein condensate $[18,19]$. In most cases, the formation of molecules is deduced from an observed reduction in the number of trapped atoms as a function of the wavelength of the photoassociation laser. In some cases, the produced molecules have also been detected directly $[13,16,17]$. Formation of mixed-alkali species is also being pursued [20,21], but has turned out to be considerably more difficult. Once formed, the molecules can be trapped in the focus of an intense laser beam [22]. In their triplet state, the molecules can also be trapped in an inhomogeneous magnetic field. Recently, magnetic trapping of $2 \times 10^{5} \mathrm{Cs}_{2}$ molecules at a temperature of $50 \mu \mathrm{K}$ has been reported [23].

In the second technique molecules are cooled via collisions with a helium buffer gas in a cryogenic cell. For ${ }^{3} \mathrm{He}$, temperatures down to $250 \mathrm{mK}$ can be reached at buffer gas densities that are still sufficiently high to allow for efficient cooling. In this way, about $10^{8} \mathrm{CaH}$ molecules have been cooled and magnetically trapped at a temperature of $400 \mathrm{mK}$ [24]. This method is applicable to any paramagnetic molecule (or atom) provided that a method is available to bring the molecule into the cryogenic cell. In the case of $\mathrm{CaH}$, laser ablation of a solid precursor mounted in the cell is used. In order to obtain a truly isolated sample of cold molecules, the buffer gas needs to be rapidly pumped out. This has already been demonstrated to work for atoms [25].

In this paper the third technique, in which time-varying electric fields are used to decelerate and trap polar molecules, is described in detail. Static electric fields have been used to deflect and focus polar molecules since the 1920s. Already in the 1950s it was realized that time-varying electric fields can be used to change the longitudinal velocity of polar molecules [26-30]. Molecules having their dipole-moment oriented antiparallel to an electric field will gain Stark energy upon entering this field. The gain in Stark energy ("potential" energy) is compensated by a loss in kinetic energy. If the electric field is greatly reduced before the molecule has left the electric field the molecule will not fully regain the lost kinetic energy. This process may be repeated by letting the molecules pass through multiple pulsed electric fields. Molecules can thus be slowed down and eventually brought to a standstill. In Ref. [31] it was first experimentally demonstrated that the longitudinal velocity of polar molecules can be changed using time-varying electric fields. A beam of metastable CO was decelerated from $225 \mathrm{~m} / \mathrm{s}$ to $98 \mathrm{~m} / \mathrm{s}$ using an array of 63 pulsed electric fields. In Ref. [32] timevarying electric fields were used to manipulate the velocity of Cs atoms in a fountain via the dipole polarizability. In Ref. [33] a theoretical model was presented to describe the motion of molecules in an array of time-varying electric fields. In particular, it was shown that the phase-space density is constant during the deceleration process. This is nontrivial since this is not generally true when a Hamiltonian is explicitly dependent on time [34]. In Ref [35] ${ }^{14} \mathrm{ND}_{3}$ molecules were decelerated and loaded in an electrostatic quadrupole trap at a density of $10^{6}$ molecules $/ \mathrm{cm}^{3}$ and a temperature below $350 \mathrm{mK}$. In Ref. [36] a prototype electrostatic storage ring for neutral molecules has been demonstrated; bunches of deuterated ammonia molecules with a forward velocity of around $100 \mathrm{~m} / \mathrm{s}$ at a translational temperature of $10 \mathrm{mK}$ were trapped in a hexapole torus. Here we report trapping of ${ }^{14} \mathrm{ND}_{3}$ and ${ }^{15} \mathrm{ND}_{3}$ molecules in a quadrupole trap at a density on the order of $10^{7}$ molecules $/ \mathrm{cm}^{3}$. The temperature is measured to be $25 \mathrm{mK}$. The phase-space density in the trap corresponds to $2 \times 10^{-13}$, about 50 times less than the phasespace density of the initial beam.

This paper is organized as follows. In Sec. II a model describing the longitudinal and transverse motion in the decelerator is outlined. From this model, the distribution of molecules in phase-space that is transmitted by the decelerator is derived. In Sec. III matching of the phase-space distribution throughout the apparatus is described. Experiments on the deceleration of a beam of ${ }^{14} \mathrm{ND}_{3}$ molecules are presented 


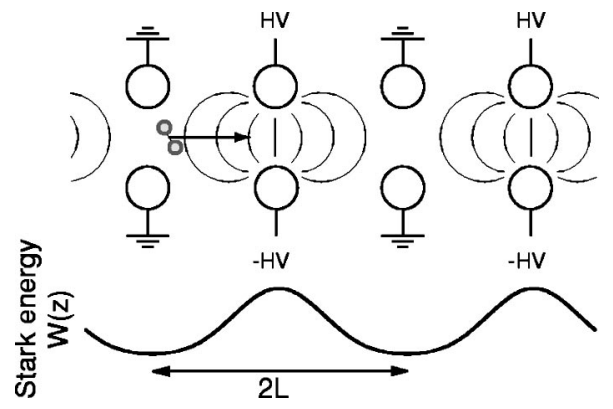

FIG. 1. Scheme of the Stark decelerator, together with the Stark energy of a molecule as a function of position $z$ along the molecular beam axis.

in Sec. IV, supporting the model. In Sec. V simultaneous deceleration of different molecules is discussed and experiments on simultaneous deceleration of the ${ }^{14} \mathrm{NH}_{3},{ }^{14} \mathrm{ND}_{3}$, and ${ }^{15} \mathrm{ND}_{3}$ ammonia isotopomers are presented. The properties of the electrostatic trap and the method used to load the trap, are discussed in Sec. VI. In Sec. VII experimental results on the loading of the decelerated beam of ammonia molecules into the trap are presented. The actual trapping of the molecules, including the experimental study of oscillations in the trap, is described in Sec. VIII. Conclusions and future prospects are given in Sec. IX.

\section{MOTION IN THE STARK DECELERATOR}

\section{A. Longitudinal motion}

There is a strong similarity between deceleration of polar molecules in a Stark decelerator and the acceleration of charged particles in a linear accelerator. In a charged particle accelerator an electric field acts on the charge of a particle, whereas in a neutral particle accelerator an electric field gradient acts on the dipole moment of a particle. With a suitable translation all concepts that are used throughout for charged particle accelerators can be used for neutral particle accelerators as well. In particular, the principle of "phase-stability," i.e., the principle that is used to keep a bunch of particles together throughout the acceleration process, can be exploited. Phase stability was independently discovered by Veksler [37] and McMillan [38] and forms the basis of all modern charged particle accelerators. In this section it will be shown how phase stability can be applied to the deceleration of polar molecules.

Consider an array of electric field stages separated by a distance $L$, as shown in Fig. 1. Each stage consists of two parallel cylindrical metal rods with radius $r$, centered a distance $2 r+d$ apart. One of the rods is connected to a positive and the other to a negative switchable high-voltage power supply. Alternating stages are connected to each other. When a molecule in a quantum state with a positive Stark effect (a so-called low-field seeker) moves through the array of electric field stages as indicated in Fig. 1, it will gain Stark energy. This gain in potential energy is compensated by a loss in kinetic energy. If the electric field is abruptly switched off, the molecule will keep its instantaneous velocity. If, simultaneously, the electric field of the next stage is switched on, the process will repeat itself. In Fig. 1 the potential energy of the molecule, $W(z)$, is depicted as a function of its position $z$ along the beam axis. The energy a molecule loses per stage depends on its position at the time that the fields are being switched. In analogy with concepts used in charged particle accelerators, this position is expressed in terms of a "phase-angle" $\phi$ that has a periodicity of $2 L$. Molecules that are in maximum electric field just prior to the time at which the fields are being switched are assigned a phase angle of $\phi=90^{\circ}$.

First the situation where the electric fields are switched at equal time intervals $\Delta T$, is discussed. Let us consider a molecule at a phase $\phi=0^{\circ}$ and with a velocity that matches the frequency of the electric fields, i.e., a molecule that travels exactly the distance $L$ in a time interval $\Delta T$. This molecule will be referred to as the "synchronous" molecule. Its phase and velocity are indicated as the equilibrium phase $\phi_{0}$ and the equilibrium velocity $v_{0}$, respectively. It is readily seen that (i) the phase and velocity of the synchronous molecule remains unchanged, and (ii) that molecules with a slightly different phase or velocity will experience an automatic correction towards these equilibrium values. A molecule with a phase slightly larger than $\phi_{0}$ and a velocity equal to $v_{0}$, for instance, will lose more energy per stage than the synchronous molecule. It will thus be slowed down relative to the synchronous molecule and consequently its phase will get smaller, until it lags behind. At this point, the situation is reversed and it will lose less energy than the synchronous molecule will, etc. This argument shows that molecules with a slightly different phase from $\phi_{0}$ and/or a slightly different velocity from $v_{0}$ will oscillate with both phase and velocity around the equilibrium values; the molecules are trapped in a potential well travelling at the velocity of the synchronous molecule.

In order to decelerate the molecules one has to lower the velocity of the potential well, by gradually increasing the time intervals $\Delta T$ after which the electric fields are being switched. The synchronous molecule will still travel a distance $L$ in the interval $\Delta T$, but $\phi_{0}$ will now be different from zero. By definition, the synchronous molecule is always at the same position when the fields are being switched $\left(\phi_{0}\right.$ remains constant); it will achieve this by losing exactly the required kinetic energy per stage. Again, the phase and velocity of a nonsynchronous molecule will oscillate around those of the decelerated synchronous molecule.

The kinetic energy lost by the synchronous molecule per stage $\Delta K\left(\phi_{0}\right)$, is given by $W\left(\phi_{0}\right)-W\left(\phi_{0}+\pi\right)$. It is convenient to express $W(\phi)$ as a Fourier series. In the expression for $\Delta K\left(\phi_{0}\right)$ all the even terms cancel, yielding

$$
\Delta K\left(\phi_{0}\right)=2 a_{1} \sin \left(\phi_{0}\right)+2 a_{3} \sin \left(3 \phi_{0}\right)+\cdots .
$$

When adjacent electric field stages are not too far apart, i.e., $L \sim 2 r+d, \Delta K\left(\phi_{0}\right)$ is predominantly determined by the first term. As mentioned above, the phase is only defined at the moment at which the fields are being switched. To be able to mathematically describe the motion of the molecules through the Stark decelerator, a description in terms of continuous variables is needed. For a description of the motion 
of a nonsynchronous molecule relative to the motion of the synchronous molecule, the instantaneous difference in phase, $\Delta \phi=\phi-\phi_{0}$, and velocity, $\Delta v=v-v_{0}$, is introduced. One can regard the lost kinetic energy per stage of the synchronous molecule to originate from a continuously acting average force $\bar{F}\left(\phi_{0}\right)=-\Delta K\left(\phi_{0}\right) / L$. This approximation can be made provided that the deceleration rate, i.e., the amount by which the velocity of the synchronous molecule is reduced in a given stage relative to $v_{0}$, is small. When $\Delta v \ll v_{0}$, the average force on a nonsynchronous molecule can be written as $\bar{F}\left(\phi_{0}+\Delta \phi\right) \simeq-\Delta K\left(\phi_{0}+\Delta \phi\right) / L$. The equation describing the motion of the nonsynchronous molecule relative to the motion of the synchronous molecule is thus given by

$$
\frac{m L}{\pi} \frac{d^{2} \Delta \phi}{d t^{2}}+\frac{2 a_{1}}{L}\left[\sin \left(\phi_{0}+\Delta \phi\right)-\sin \left(\phi_{0}\right)\right]=0,
$$

with $m$ the mass of the molecule. This is analogous to the equation for a pendulum driven by a constant torque. When $\Delta \phi$ is small, $\sin \left(\phi_{0}+\Delta \phi\right) \simeq \sin \phi_{0}+\Delta \phi \cos \phi_{0}$ and the equation of motion becomes

$$
\frac{m L}{\pi} \frac{d^{2} \Delta \phi}{d t^{2}}+\frac{2 a_{1}}{L}\left(\Delta \phi \cos \phi_{0}\right)=0 .
$$

In Fig. 2 a numerical integration of Eq. (2) is shown for various equilibrium phases $\phi_{0}$, with parameters as used in the experiment on ${ }^{14} \mathrm{ND}_{3}$, to be described later. The solid curves are lines of constant energy, and indicate trajectories that molecules will follow. The inner curves are nearly elliptical, corresponding to a nearly linear restoring force on the molecules, as can be seen from Eq. (3). For ${ }^{14} \mathrm{ND}_{3}$ the longitudinal oscillation frequency, given by

$$
\omega_{z} / 2 \pi=\sqrt{\frac{a_{1}}{2 \pi m L^{2}} \cos \phi_{0}},
$$

is approximately $1 \mathrm{kHz}$ for $\phi_{0}=70^{\circ}$. Further outward, the restoring force is less than linear and the oscillation frequency is lowered, approaching zero on the separatrix (thick curve). Beyond the separatrix the total energy of the molecules will be larger than the effective potential well and the trajectories of the molecules are unbound. The area inside the separatrix ("bucket") is the longitudinal acceptance of the decelerator. It is seen that this area is larger for smaller values of $\phi_{0}$. The deceleration per stage is given by Eq. (1) and increases when $\phi_{0}$ is increased from 0 to $\frac{1}{2} \pi$. Since both a large acceptance and efficient deceleration are desirable there is a trade-off between the two.

The phase-stability diagrams showing the longitudinal acceptance of the Stark decelerator are very similar to those used to describe charged particle accelerators (see, for instance, Fig. 2.20 of Ref. [39]). There is an important difference, however. In a charged particle accelerator energy is added to the particles at a certain position, while the amount of energy that is added depends on the time at which they arrive at this position. In the Stark decelerator energy is added to the molecules at a certain time, while the amount of
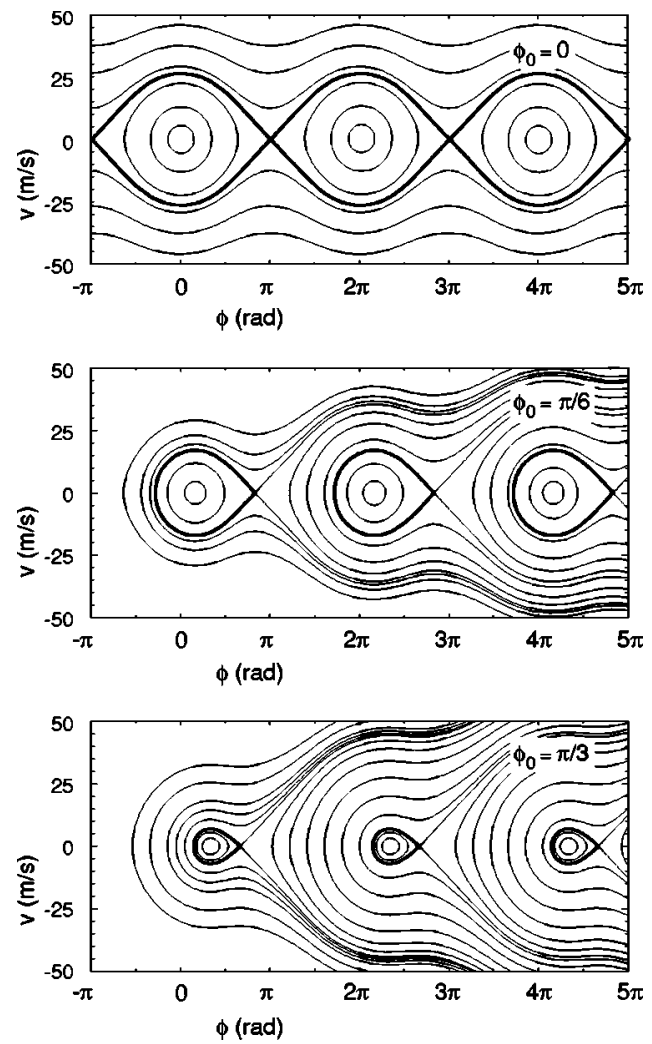

FIG. 2. Phase-stability diagrams for various values of $\phi_{0}$, obtained via numerical integration of Eq. (2) with parameters as used in the experiment for ${ }^{14} \mathrm{ND}_{3}$. In the experiment, a difference in the phase angle of $2 \pi$ corresponds to a distance of $11 \mathrm{~mm}$.

energy that is added depends on their position at that time. Therefore, while in charged particle accelerators energy and time are conjugate variables, in the Stark decelerator this role is played by velocity (energy divided by velocity) and position (time multiplied by velocity). As a consequence, while the energy spread in the laboratory frame remains constant in a charged particle accelerator, the velocity spread in the laboratory frame remains constant in the Stark decelerator.

Phase stability ensures that the phase-space density remains constant during the deceleration process. In addition, it makes the deceleration insensitive to small deviations from the designed electric field in the decelerator, caused by, for instance, misalignments of the electrodes or fluctuations in the applied voltages. Small deviations from the anticipated electric field will make the phase $\phi_{0}$, assumed to be constant throughout the decelerator, differ per stage. This will lead to a slightly modified acceptance area, with some blurring in the region of the separatrix. The final velocity, however, is determined by the time sequence only and will not be altered.

In Ref. [33] this model was tested using metastable CO molecules. In this experiment the molecules were decelerated to $200 \mathrm{~m} / \mathrm{s}$, such that the deceleration rate and the relative velocity spread $\left(\Delta v_{z} / v_{z}\right)$ was kept low. The model was found to accurately describe the deceleration process. In this article data are presented where a beam of ammonia molecules is decelerated to much lower velocities, where these approximations no longer hold. In addition, these experi- 
ments are sensitive to the effects of the transverse motion in the decelerator.

\section{B. Transverse motion}

For the array of electrodes schematically depicted in Fig. 1 the electric field close to the electrodes is higher than that on the molecular beam axis. Therefore, molecules in lowfield seeking states will experience a force focusing them towards the molecular beam axis. This focusing occurs only in the plane perpendicular to the electrodes, i.e., in the plane of the figure. In order to focus the molecules in both transverse directions, the electrode pairs that make up one deceleration stage are alternately positioned horizontally and vertically.

In Fig. 3 the electric field in the decelerator is shown for two mutually perpendicular planes. Both planes that are shown have the molecular beam axis in common. The electric fields are calculated using a finite-element program [40], with parameters as in the experiment. The two opposing rods that make up one deceleration stage have a radius $r$ of 1.5 $\mathrm{mm}$ and are spaced a distance $d$ of $2 \mathrm{~mm}$ apart. Two adjacent deceleration stages are spaced a distance $L$ of $5.5 \mathrm{~mm}$ apart. With a maximum voltage of $+10 \mathrm{kV}$ and $-10 \mathrm{kV}$ on the electrodes the maximum electric field on the molecular beam axis is $90 \mathrm{kV} / \mathrm{cm}$. Close to the electrodes at high voltage the electric field is $120 \mathrm{kV} / \mathrm{cm}$. The electric fields shown in Fig. 3 are calculated for the situation with the vertical electrodes (front part) at ground potential and with the horizontal electrodes (partly hidden) at high voltage. Therefore, molecules in low-field-seeking states are focused in the plane perpendicular to the electrodes at high voltage, as shown in the upper part of the figure. In the other direction the electric field is (nearly) constant and therefore there will be no focusing or defocusing in this direction. During the deceleration process the molecules pass through electric field stages that are alternately positioned horizontally and vertically, and are therefore focused in either direction.

As can be seen from Fig. 3, the force experienced by a molecule depends on its position $z$ in the decelerator. In addition, it depends on the time sequence of the high-voltage pulses that are applied. The molecule is strongly focused when in between the electrodes at high voltage but much less so further away from these electrodes. Therefore, the trajectories will generally be complicated. When the focusing is not too strong the $z$ dependence of this force can be eliminated by introducing an averaged force. The transverse motion throughout the decelerator can then be described using this averaged force, which will now depend on the phase angle $\phi$ only. The motion of the molecules in the transverse potential well is characterized by a transverse angular oscillation frequency $\omega_{t}(\phi)$. The condition that the focusing is not too strong can be formulated as $2 \pi / \omega_{t} \gg 2 L / v_{z}$, i.e., that the time of transverse oscillation is much larger than the time it takes for the molecules to traverse one period (two stages) of the decelerator. Molecules with a longitudinal velocity close to the velocity of the synchronous molecules will traverse a distance $L$ during $\Delta T$, the time interval after which the voltages are being switched. The force needs to be averaged over $2 L$
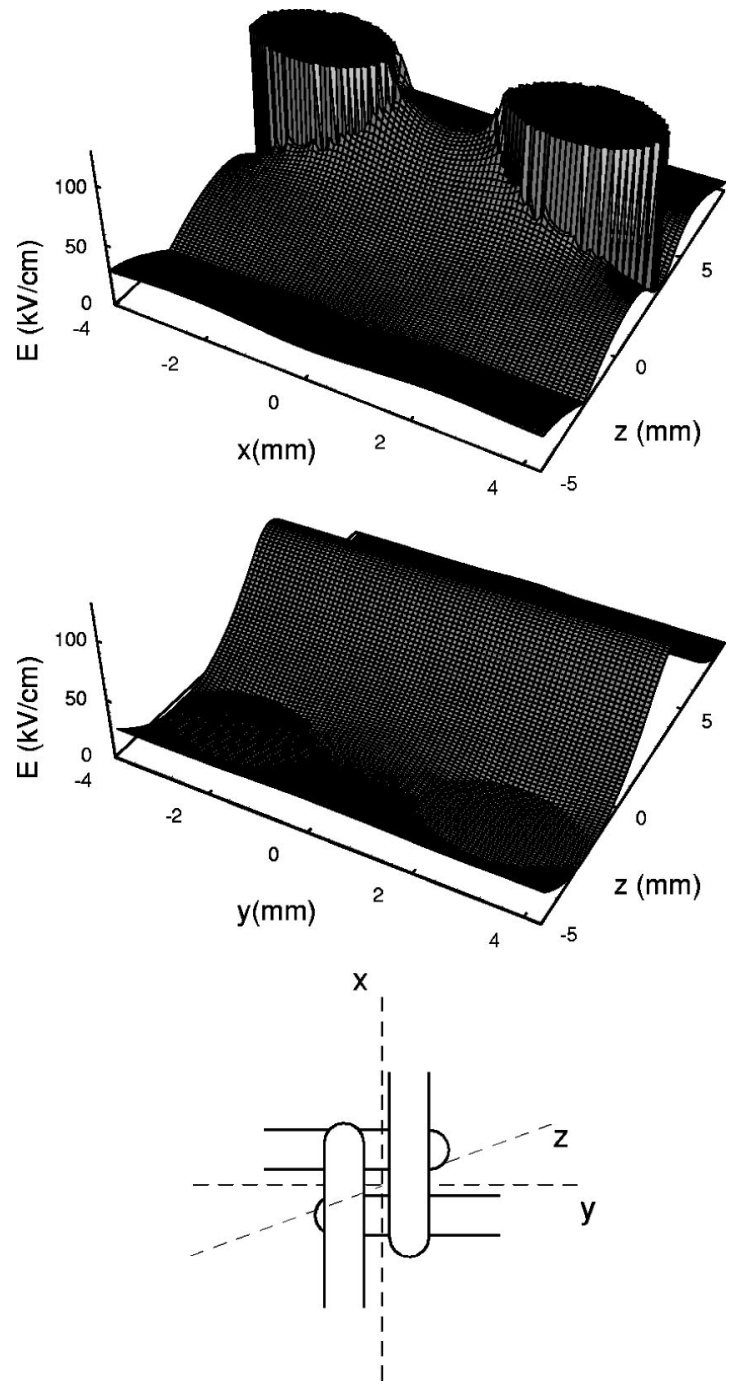

FIG. 3. The electric field in two mutually perpendicular planes that have the molecular beam axis in common, together with a schematic view of two adjacent electric field stages. The two vertical rods are at ground potential, while the horizontal rods are at $+10 \mathrm{kV}$ and $-10 \mathrm{kV}$.

$$
\bar{F}_{t}(\phi)=\int_{\phi L / \pi}^{(\phi+2 \pi) L / \pi F_{t}(z)} \frac{2 L}{2 L} d z .
$$

Close to the molecular beam axis, the average force is linearly dependent on the displacement from this axis. The solid curve in Fig. 4 shows the resulting force constant for ${ }^{14} \mathrm{ND}_{3} \quad\left(1 \mathrm{aN} / \mathrm{m} \simeq 0.05 \mathrm{~cm}^{-1} / \mathrm{mm}^{2}\right)$. The dashed curve shows the maximum depth of the transverse potential well, i.e., the depth $1 \mathrm{~mm}$ away from the molecular beam axis. Since the linear dependence of the force on the displacement holds rather well throughout, the curves are almost identical. For $\phi=70^{\circ}$ the transverse well is $\sim 0.014 \mathrm{~cm}^{-1}$, or $\sim 20 \mathrm{mK}$, deep. Molecules with a maximum transverse velocity of $4 \mathrm{~m} / \mathrm{s}$ will, therefore, still be accepted in the deceleration process. The frequency of transverse oscillation, $\omega_{t} / 2 \pi$, is approximately $800 \mathrm{~Hz}$.

Generally a molecule will oscillate between a minimum and a maximum phase and it will, therefore, experience a 


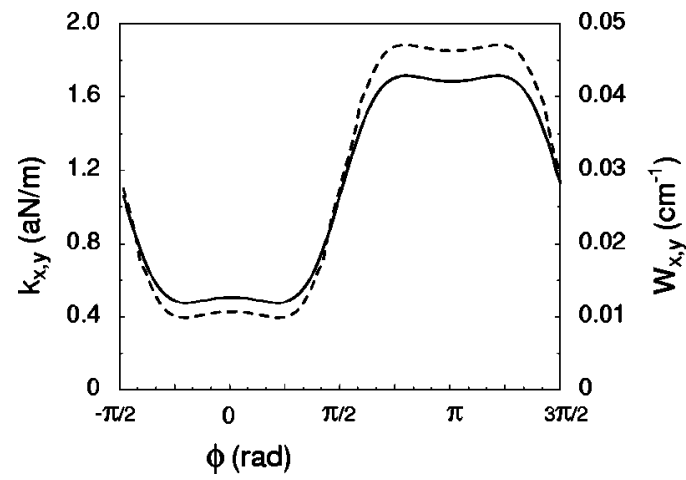

FIG. 4. The average force constant (close to the molecular beam axis) for ${ }^{14} \mathrm{ND}_{3}$ as a function of $\phi$ (solid curve) together with the maximum depth of the transverse potential well (dashed curve), i.e., the depth $1 \mathrm{~mm}$ away from the molecular beam axis.

different (averaged) transverse force along its path. The coupling between the longitudinal motion and the transverse motion may result in parametric amplification of the transverse oscillation. This effect might be expected to be strong, as the frequencies involved are rather similar. On the other hand, as the deceleration process only lasts $2-3$ oscillation periods, parametric amplification is not expected to be important, as confirmed by Monte Carlo simulations. The maximum transverse velocity that will be accepted is determined by the minimum depth of the transverse potential well that the molecules pass through. This implies that the maximum transverse acceptance is obtained for molecules with a phase around that of the synchronous molecule.

Since a maximum of the electric field strength cannot be produced in free space [41], molecules in high-field-seeking states cannot be simultaneously focused in both transverse directions. It is possible, however, to produce a field that has a maximum in one direction and a minimum in the other direction. By alternating the orientation of these fields it is possible to obtain net focusing ("alternate gradient focusing") for molecules in high-field-seeking states in either direction [28]. When combined with the use of time varying electric fields, an alternate gradient decelerator can be constructed [42].

\section{PHASE-SPACE MATCHING}

As shown in the preceding sections the area in phase space remains constant throughout the deceleration process. This in itself is not enough. When the phase-space distribution of the molecular beam ("emittance") does not match the phase-space acceptance of the decelerator (where acceptance is used to designate the shape of the curves of constant energy rather than the maximum curve of constant energy) the area in phase-space sampled by the molecules will be much larger. (see for instance Fig. 3 in Ref. [33]). Although the area remains constant in this case, the original distribution is in practice irretrievable, and the effective area in phase space is increased, i.e., the effective (time-averaged) phase-space density is decreased. In order to avoid this, one needs to shape the emittance properly, a process known as "phasespace matching" [34]. The setup required to decelerate and

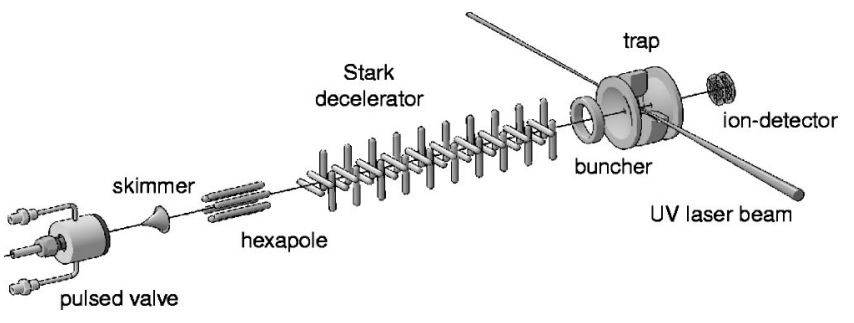

FIG. 5. Schematic view of the experimental setup. A beam of ammonia molecules is decelerated in a $35-\mathrm{cm}$-long decelerator and loaded into an electrostatic trap. In order to detect the ammonia molecules a pulsed laser is focused inside the trap. The resulting ions are extracted and counted using an ion detector.

trap a molecular beam therefore consists of essentially five parts; (i) the molecular beam source, (ii) a device to match the beam to the input of the decelerator, (iii) the decelerator, (iv) a device to match the beam exiting the decelerator to the trap, and, (v) the trap itself. In this way a 6D area in phase space is imaged from the source region of the molecular beam onto the trap.

\section{DECELERATION EXPERIMENTS}

The experimental setup, schematically depicted in Fig. 5, consists of a compact molecular beam machine, with two differentially pumped vacuum chambers. The source chamber and decelerator chamber are pumped by a $300 \mathrm{l} / \mathrm{s}$ and a $240 \mathrm{l} / \mathrm{s}$ turbo pump, respectively. A pulsed beam of ammonia is formed by expanding a mixture of less than $1 \%$ ammonia in xenon through a modified solenoid valve with a $0.8 \mathrm{~mm}$ diameter opening into vacuum. The solenoid valve (General Valves series 9) is modified such that it can be operated down to liquid nitrogen temperatures. In these experiments the valve housing is cooled to $200 \mathrm{~K}$, such that the vapor pressure of ammonia remains sufficiently high. The stagnation pressure is typically about $1.5 \mathrm{~atm}$. The valve opens for a duration of $80 \mu \mathrm{s}$. The experiments run at $10 \mathrm{~Hz}$, and under operating conditions the pressure in the source chamber and the decelerator chamber is typically $4 \times 10^{-6}$ and 2 $\times 10^{-8}$ Torr, respectively. The peak intensity of the beam is limited by the pumping capacity; at higher pressures in the source chamber the beam collapses.

The various ammonia isotopomers have different possible symmetries for the nuclear spin wave functions of the identical H/D nuclei. For each rovibrational level of the ammonia molecule a combination with a nuclear spin wave function of one specific symmetry is allowed. In the expansion, the molecules are adiabatically cooled, and only the lowest rotational levels in the vibrational and electronic ground state are populated in the beam. In this cooling process the symmetry of the nuclear spin wave function is preserved. Therefore, the ratio of the populations in the levels having different symmetries of the nuclear spin wave function is the same in the molecular beam as in the original sample. For $\mathrm{NH}_{3}$ and $\mathrm{ND}_{3}$ this implies that roughly $60 \%$ of the molecules in the beam reside in the $|J, K\rangle=|1,1\rangle$ level, the ground-state level for molecules having a nuclear spin wave function of $E$ symmetry. For historical reasons, levels of $E$ symmetry in $\mathrm{NH}_{3}$ and 


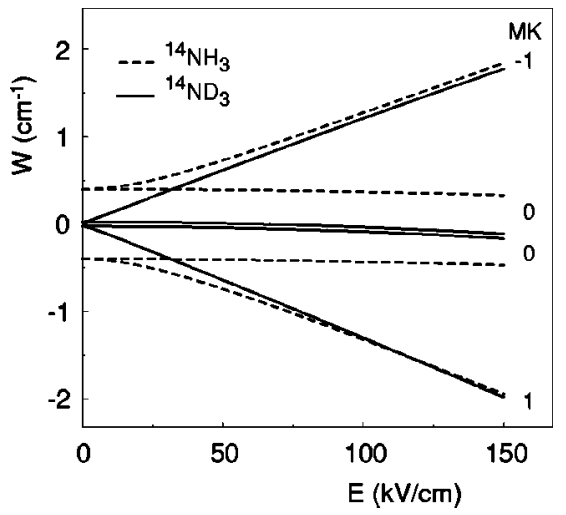

FIG. 6. Stark shift of the $|J, K\rangle=|1,1\rangle$ level of ${ }^{14} \mathrm{NH}_{3}$ and ${ }^{14} \mathrm{ND}_{3}$ ammonia molecules in an electric field of up to $150 \mathrm{kV} / \mathrm{cm}$. On this scale the Stark shifts of ${ }^{14} \mathrm{ND}_{3}$ and ${ }^{15} \mathrm{ND}_{3}$ (not shown) are identical.

$\mathrm{ND}_{3}$ are denoted as para levels. The only other possible symmetry of the nuclear spin wave function in $\mathrm{NH}_{3}$ is $A_{1}$ symmetry, and levels having this symmetry are denoted as ortho levels. For $\mathrm{ND}_{3}$ both $A_{1}$ and $A_{2}$ symmetry is possible; levels having either one of this symmetry are denoted as ortho levels.

In zero electric field, the $|J, K\rangle=|1,1\rangle$ level of $\mathrm{NH}_{3}$ and $\mathrm{ND}_{3}$ is split due to tunneling in a symmetric and in an antisymmetric component. Each of these components is further split due to hyperfine interactions. The hyperfine splitting is less than $2 \mathrm{MHz}$ for ${ }^{14} \mathrm{ND}_{3}$ and less than $0.5 \mathrm{MHz}$ for ${ }^{15} \mathrm{ND}_{3}$ [43], and will be neglected in the following. Upon applying an electric field, the symmetric and the antisymmetric components interact and repel each other, leading to four levels, as shown in Fig. 6. Throughout this work molecules in the $|J, M K\rangle=|1,-1\rangle$ state are used, which is the only low-fieldseeking state populated in the molecular beam; roughly $20 \%$ of the ammonia molecules in the beam are in this state. At low electric fields the Stark shift of this state is quadratic. When the Stark interaction becomes large relative to the zero-field splitting, the Stark shift is linear.

It is essential that the molecules remain in the low-fieldseeking state throughout the decelerator. If the molecules would come in zero electric field, they might project onto the $|J, M K\rangle=|1,0\rangle$ state, and they would be lost for the deceleration process. These so-called Majorana transitions are avoided by assuring that the electric field never drops below a certain minimum value [44]. Furthermore, the rapid switching of the electric fields generates rf radiation that can induce transitions between these states in nonzero fields. To avoid these transitions, the minimum electric field needs to be kept sufficiently high that the Stark splitting is larger than that in the highest-frequency component of the rf radiation. Molecules moving through the decelerator never experience an electric field below $300 \mathrm{~V} / \mathrm{cm}$, and no sign of (loss due to) these transitions has been observed. It should be noted, however, that the rapid switching of electric fields is expected to scramble the various hyperfine levels of the low-fieldseeking component.

The average velocity of the molecular beam is around 285 $\mathrm{m} / \mathrm{s}\left(E_{k i n}=68 \mathrm{~cm}^{-1}\right)$. This is slightly higher than expected
[45], indicating that the translational velocity of the ammonia molecules is not fully equilibrated with the carrier gas. The velocity spread of the beam [full width at half maximum (FWHM)] is $20 \%$, corresponding to a translational temperature of $1.6 \mathrm{~K}$. The average velocity and the translational temperature are averages over the $80 \mu$ s duration of the gas pulse. The beam passes through a 1.0-mm-diameter skimmer into a second vacuum chamber and then flies into a $5-\mathrm{cm}$ long pulsed hexapole that acts as a positive lens for molecules in low-field-seeking states. After exiting the hexapole the molecules enter the $35-\mathrm{cm}$-long decelerator. The decelerator consists of an array of 64 deceleration stages. Each deceleration stage is formed by two parallel 3-mm-diameter cylindrical rods, spaced $2 \mathrm{~mm}$ apart. The rods are made from hardened steel and are highly polished. The two opposing rods are simultaneously switched by two independent highvoltage switches to a maximum of $+10 \mathrm{kV}$ and $-10 \mathrm{kV}$. All horizontal and vertical stages are electronically connected requiring a total of four independent high-voltage switches (Behlke Electronic GmbH, HTS-151-03-GSM). The switches are triggered by a programmable delay generator running at a clock frequency of $100 \mathrm{MHz}$. The time sequences that are used are generated by the same program that is used for the Monte Carlo simulations (vide infra).

It is advantageous to operate the decelerator at the highest possible electric fields, since this will increase the acceptance of the decelerator. The maximum obtainable electric field strength is limited by field electron emission at the electrode surfaces. It is assumed that this will take place at field strengths over $\sim 10^{4} \mathrm{kV} / \mathrm{cm}$ [46], in principle allowing a voltage difference of $2000 \mathrm{kV}$ over the $2 \mathrm{~mm}$ gap between the electrodes. In practice, however, electrical breakdown will take place at much lower voltage differences due to field enhancement at microfeatures associated with the intrinsic microscopic roughness of the electrode surfaces. Breakdown events need not be disastrous as long as the energy that is dissipated in the gap is kept low. In fact, since discharges will locally melt the surface, these events will reduce the roughness of the surface, allowing the gap to withstand a higher voltage difference after the event. Since in the decelerator the electric fields are switched rapidly (within $200 \mathrm{~ns}$ ), the resistance between the switches and the electrodes needs to be small $(1 \mathrm{k} \Omega)$. Under these conditions it is likely that a discharge will permanently damage the surface, instead of improving it. Therefore, before operating the decelerator with time varying electric fields, a constant electric field is used for high-voltage conditioning. The voltage difference is slowly increased while the current of the induced discharges is being limited. This procedure allows for an increase of the maximum attainable voltage difference between the electrodes without sparking. Typically, the voltage difference is increased by $5 \mathrm{kV} / \mathrm{h}$ while the current is limited to $1 \mu \mathrm{A}$. This procedure is repeated every time the decelerator has been exposed to air. After this treatment, the decelerator is operated at a voltage difference $25 \%$ below the maximum voltage difference sustainable during the high-voltage conditioning. In this way the decelerator could be operated routinely at $+10 \mathrm{kV}$ and $-10 \mathrm{kV}$, corresponding to a maximum electric field of $120 \mathrm{kV} / \mathrm{cm}$. It is believed that at 
present the maximum electric field is limited by currents running over the surface of the aluminum-oxide rings that are used for suspension of the decelerator. By improving the mounting of the decelerator, it is expected that the electric fields can be increased to $200 \mathrm{kV} / \mathrm{cm}$ or more.

In all experiments presented here, the decelerator is operated at voltages of $+10 \mathrm{kV}$ and $-10 \mathrm{kV}$, leading to a maximum electric field on the molecular beam axis of 90 $\mathrm{kV} / \mathrm{cm}$. In this field, the $|J, K M\rangle=|1,-1\rangle$ state is shifted by $1.1 \mathrm{~cm}^{-1}$ relative to zero electric field for ${ }^{14} \mathrm{ND}_{3}$ and ${ }^{15} \mathrm{ND}_{3}$, and by $0.8 \mathrm{~cm}^{-1}$ for ${ }^{14} \mathrm{NH}_{3}$ (see Fig. 6). In most of the experiments, a phase angle $\phi_{0}=70^{\circ}$ is used. With 64 deceleration stages ${ }^{14} \mathrm{ND}_{3}$ and ${ }^{15} \mathrm{ND}_{3}$ are then slowed down to $15 \mathrm{~m} / \mathrm{s}$, while ${ }^{14} \mathrm{NH}_{3}$ is decelerated to $\sim 100 \mathrm{~m} / \mathrm{s}$, when starting from $255 \mathrm{~m} / \mathrm{s}$. With these settings, the $6 \mathrm{D}$ acceptance area of the decelerator is approximately $[1.5 \mathrm{~mm} \times 8 \mathrm{~m} / \mathrm{s}$ ] $\times[2 \mathrm{~mm} \times 8 \mathrm{~m} / \mathrm{s}]^{2}$. The longitudinal emittance of the beam, typically $[25 \mathrm{~mm} \times 60 \mathrm{~m} / \mathrm{s}]$, is much larger than the longitudinal acceptance of the decelerator, and there is nothing to be gained by changing the shape of the emittance in this direction. The transverse emittance of the beam, typically $[1 \mathrm{~mm} \times 20 \mathrm{~m} / \mathrm{s}]^{2}$, is better matched to the transverse acceptance of the decelerator when it is expanded spatially while the velocity distribution is compressed. This is achieved by using a short hexapole to make a 1:2 image of the beam exiting the skimmer onto the entrance of the decelerator.

The hexapole consists of 3-mm-diameter rods placed equidistantly on the outside of a circle with a $3 \mathrm{~mm}$ radius. The rods of the hexapole are alternatingly at ground potential and at $10 \mathrm{kV}$, creating an electric field that is cylindrically symmetric close to the molecular beam axis. Generally, the field in an $n$ pole is proportional to $r^{(n / 2)-1}$ [47], yielding a quadratic dependence of the electric field on $r$ in our case. Molecules with a linear positive Stark effect will thus experience a linear force towards the molecular beam axis. In free flight from the skimmer to the hexapole the beam spreads out in the transverse spatial direction, while the transverse velocity remains unchanged. This creates an elongated distribution in the corresponding phase space, tilted with respect to the spatial coordinate. When switching on the field of the hexapole, this distribution will start to rotate in phase space. When the hexapole is switched off at the appropriate time, the beam will refocus in free flight from the hexapole to the decelerator, forming an image of the beam (at the skimmer) onto (the entrance of) the decelerator. By placing the hexapole at the right position, or, alternatively, by using a long hexapole that is pulsed at the right time, a 1:2 image of the beam can be formed. Due to the zero-field splitting, molecules close to the molecular beam axis will be less well focused, and the image will not be perfect [48]. Almost a factor of 4 increase in phase-space density is expected at the exit of the decelerator compared to the situation where the hexapole is not used. A clear increase has indeed been observed. In most of the data presented here the hexapole could not be used, however, due to problems with electrical discharges. In Fig. 7 the density of ${ }^{14} \mathrm{ND}_{3}$ molecules $24.5 \mathrm{~mm}$ behind the decelerator, i.e., at the center of the trap, is shown as a function of time after the start of the time sequence.

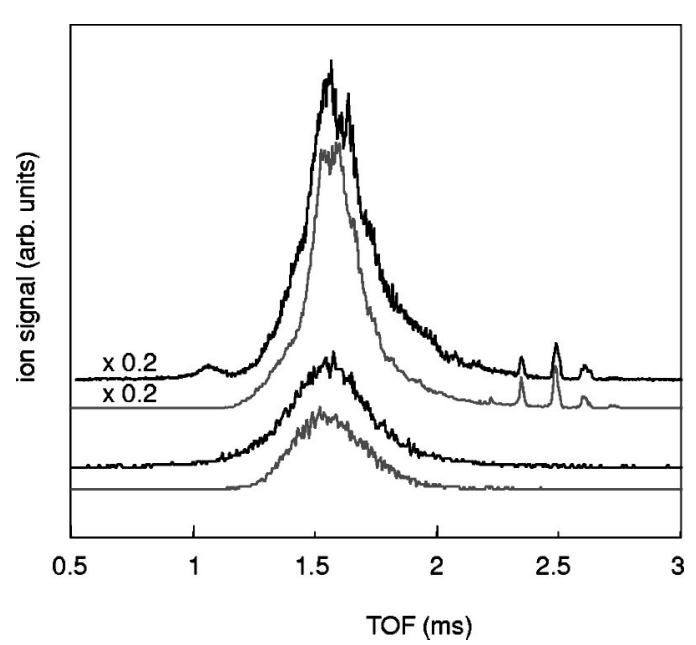

FIG. 7. TOF profiles for ${ }^{14} \mathrm{ND}_{3}$ molecules recorded $24.5 \mathrm{~mm}$ behind the decelerator as a function of time after the start of the time sequence. Using $(2+1)$-REMPI with a pulsed laser around $317 \mathrm{~nm},{ }^{14} \mathrm{ND}_{3}$ molecules in the $|J, K\rangle=|1,1\rangle$ upper inversion level are selectively detected. The lower curve (bold) shows the original beam when the decelerator is off. The upper curve (bold) shows the time-of-flight (TOF) profile when the beam is decelerated from $271.5 \mathrm{~m} / \mathrm{s}$ to $91.8 \mathrm{~m} / \mathrm{s}$. Bunches of slow molecules exit the decelerator at later times, arriving in the detection region around $2.5 \mathrm{~ms}$ after the start of the time sequence. The gray curves are the result of a Monte Carlo simulation of the experiment. The signal intensity typically increases with a factor of 14 due to transverse focusing when the decelerator is switched on; the signal of the slow molecules is larger than that of the original beam.

Using a pulsed tunable UV laser, ${ }^{14} \mathrm{ND}_{3}$ molecules in the $|J, K\rangle=|1,1\rangle$ upper component of the inversion doublet are selectively ionized in a $(2+1)$ resonance-enhanced multiphoton ionization (REMPI) scheme. The laser radiation, about $10 \mathrm{~mJ}$ of energy around $317 \mathrm{~nm}$ in a 5-ns-duration pulse, is focused inside the trap using a lens with a focal length of $75 \mathrm{~cm}$. Mass-selective detection of the parent ions is performed using the trap electrodes as extraction electrodes in a Wiley-McLaren-type mass spectrometer setup. For this, a voltage of $-300 \mathrm{~V}$ is applied to the exit endcap. The field-free flight tube is kept at $-1.2 \mathrm{kV}$ and the ion signal is recorded using a microchannel plate detector placed further downstream. The ion signal is proportional to the density of neutral ammonia molecules at the center of the trap, and amounts to approximately 125 ion counts per laser pulse for the decelerated molecular beam. The lower curve in Fig. 7 shows the time of flight (TOF) profile of the original beam. The beam has an average velocity of $285 \mathrm{~m} / \mathrm{s}$ and a velocity spread of $60 \mathrm{~m} / \mathrm{s}$. The upper curve (bold) shows the TOF profile when the decelerator is used to slow down the molecules from $271.5 \mathrm{~m} / \mathrm{s}$ to $91.8 \mathrm{~m} / \mathrm{s}$. At the entrance of the decelerator the beam has an extension along $z$ of around 25 $\mathrm{mm}$ (FWHM). This is more than twice as long as the periodicity $2 L$ of the decelerator, and thus more than one bucket will be filled (see Fig. 2). Three peaks are clearly observed in the TOF profile around $2.5 \mathrm{~ms}$. The central peak originates from molecules that were at the right position near the entrance of the decelerator at the start of the time sequence, and 
that passed through all 64 deceleration stages. These molecules entered the decelerator with a velocity of $271.5 \mathrm{~m} / \mathrm{s}$ and exit the decelerator with a velocity of $91.8 \mathrm{~m} / \mathrm{s}$. The earlier peak originates from molecules that were already one period further inside the decelerator at the start of the time sequence. These molecules also entered the decelerator with a velocity of $271.5 \mathrm{~m} / \mathrm{s}$, but since they missed the last two deceleration stages they exit the decelerator with a slightly higher velocity of $102 \mathrm{~m} / \mathrm{s}$. The peak appearing at a later time in the TOF distribution originates from molecules entering the decelerator with a lower initial velocity of $268 \mathrm{~m} / \mathrm{s}$, catching up with the time sequence one period later. Throughout the decelerator they trail the central group of molecules by $11 \mathrm{~mm}$, exiting the decelerator with the same final velocity of $91.8 \mathrm{~m} / \mathrm{s}$. Due to transverse focusing of the beam, the signal intensity typically increases with a factor of 14 when the decelerator is switched on; the signal of the decelerated beam is 1.4 times as large as the signal of the original beam. It should be noted that the signal of the original beam is the signal to which all hyperfine structure levels of the upper inversion component contribute whereas only $2 / 3$ of the levels (48 of the 72 hyperfine levels, including $m$ degeneracy) contribute to the signal when the decelerator is switched on. The gray curves show the result of a 3D Monte Carlo simulation of the experiment using the calculated electric field and the Stark effect of ${ }^{14} \mathrm{ND}_{3}$ as input. The Gaussian velocity distribution that has been used as input for the Monte Carlo simulation has been adjusted to match the observed TOF profile of the original beam. With this input, the simulations are seen to quantitatively reproduce the TOF profiles recorded with the decelerator on.

In Fig. 8 the density of ${ }^{14} \mathrm{ND}_{3}$ molecules $24.5 \mathrm{~mm}$ behind the decelerator is shown as a function of time after the start of the time sequence. The TOF profiles are all recorded using a time sequence corresponding to a phase angle $\phi_{0}=70^{\circ}$. Lower final velocities are obtained by starting from lower initial velocities; final velocities of $91.8 \mathrm{~m} / \mathrm{s}, 73.5 \mathrm{~m} / \mathrm{s}, 57.9$ $\mathrm{m} / \mathrm{s}, 36 \mathrm{~m} / \mathrm{s}$, and $15 \mathrm{~m} / \mathrm{s}$ are obtained by starting from initial velocities of $271.5 \mathrm{~m} / \mathrm{s}, 265.9 \mathrm{~m} / \mathrm{s}, 261.9 \mathrm{~m} / \mathrm{s}, 258 \mathrm{~m} / \mathrm{s}$, and $256 \mathrm{~m} / \mathrm{s}$, respectively. The gray curves show the result of a one-dimensional Monte Carlo simulation of the TOF profiles. For each TOF profile, the results from the Monte Carlo simulation are shown as dots in a phase-space diagram, together with some trajectories calculated using the model presented in Sec. II A. The dots represent the position and velocity of individual molecules along the molecular beam axis at the exit of the decelerator, relative to those of the synchronous molecule. In the lower graph, the initial velocity and position at the entrance of the decelerator of the molecules that are decelerated from $256 \mathrm{~m} / \mathrm{s}$ to $15 \mathrm{~m} / \mathrm{s}$ are shown as well. The final kinetic energies of the bunches of molecules for which the TOF profiles are shown are separated by (multiples of) $0.8 \mathrm{~cm}^{-1}$. The phase-space distributions can therefore be interpreted as the phase-space distribution inside the decelerator at the 56th, 59th, 61st, 63rd, and 64th stage, for a beam that is decelerated from an initial velocity of $256 \mathrm{~m} / \mathrm{s}$ to a final velocity of $15 \mathrm{~m} / \mathrm{s}$. It is seen that the shape of the phase-space distribution of the beam at low final velocities starts to deviate from the model. The trajectories of the mol-
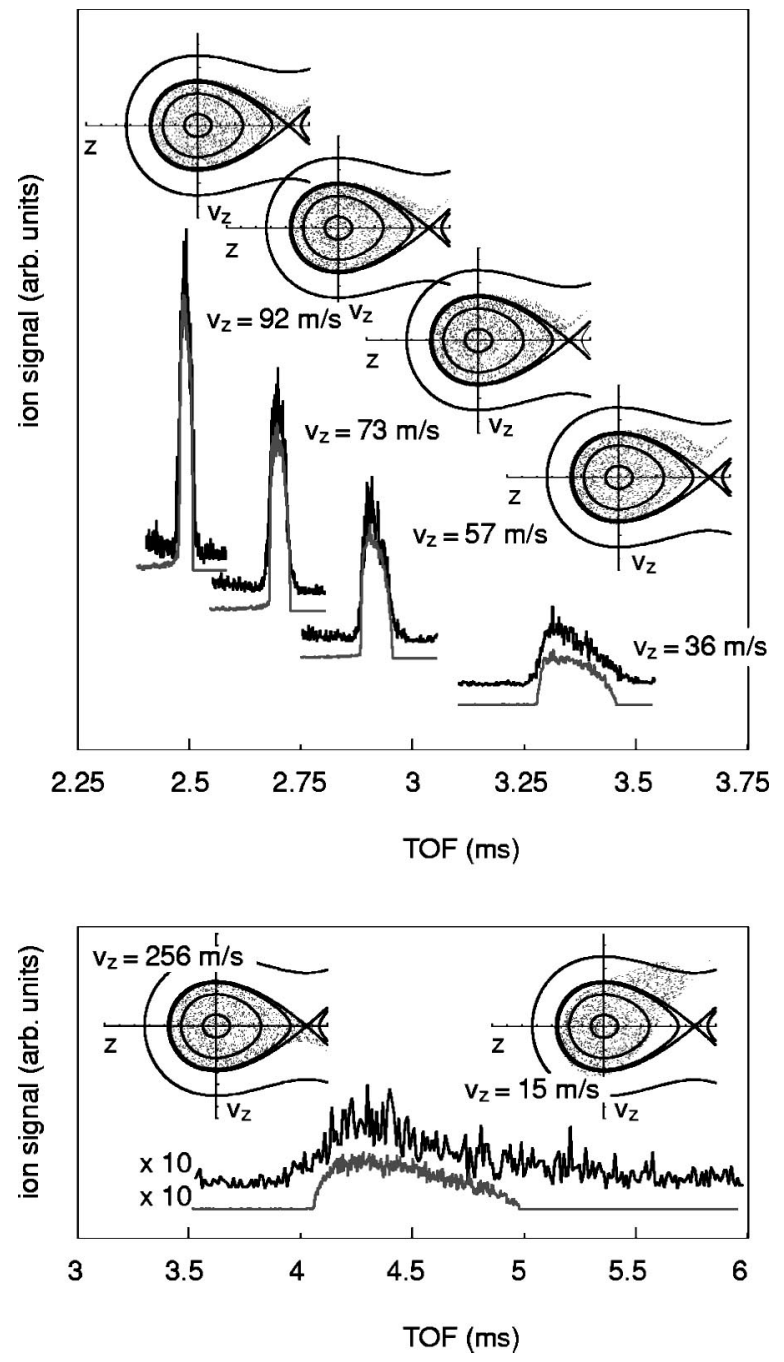

FIG. 8. TOF profiles for ${ }^{14} \mathrm{ND}_{3}$ molecules recorded $24.5 \mathrm{~mm}$ behind the decelerator as a function of time after the start of the time sequence. The TOF profiles are all recorded using a time sequence for a phase angle $\phi_{0}=70^{\circ}$, but starting from different initial velocities. The gray curves show the result of a one-dimensional Monte Carlo simulation of the TOF profiles. For each TOF profile, the results from the Monte Carlo simulation are shown as dots in a phase-space diagram, together with some trajectories calculated using the model presented in Sec. II A. The full scale of the phasespace diagrams is $-2 \mathrm{~mm}$ to $2 \mathrm{~mm}$ for the $z$ axis and $-8 \mathrm{~m} / \mathrm{s}$ to $8 \mathrm{~m} / \mathrm{s}$ for the $v_{z}$ axis.

ecules in phase space are no longer closed and the phasespace area changes "from a fishlike to a golf-club-like shape." This is known to occur also in charged particle accelerators when the acceleration rate is high (see, for instance, Ref. [49], p. 222). The phase-space distribution of the beam at $v_{z}=15 \mathrm{~m} / \mathrm{s}$ resembles a tilted ellipse, which covers the same area in phase space as the phase fish originating from the model.

Each data point in the measurements shown in Fig. 8 represents the density of ammonia molecules at the laser focus at a certain time. This signal decreases with lower forward velocity due to spreading of the package both in longitudinal and transverse directions. To eliminate the effect of 


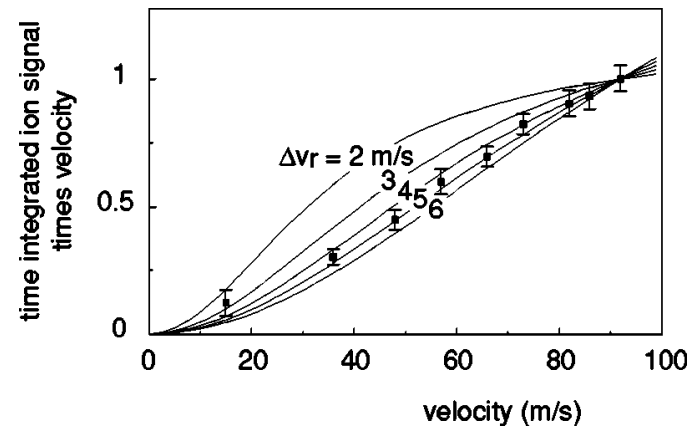

FIG. 9. Time-integrated signal of the slow molecules exiting the decelerator times their forward velocity as a function of their forward velocity, using the TOF profiles shown in Fig. 8. The measurements are normalized to the signal at $91.8 \mathrm{~m} / \mathrm{s}$. The solid curves show the expected dependence of the number of molecules passing through the laser focus on the forward velocity using transverse velocity distributions with different widths, as indicated.

the spreading in the longitudinal direction, the signal is integrated over time. The time integrated signal is proportional to the number of molecules passing through the laser focus, divided by their velocity; slow molecules contribute more to this signal than fast molecules. In Fig. 9, the time-integrated signal times the forward velocity is shown as a function of the forward velocity. The measurements are corrected for the intensity of the molecular beam at the initial velocity. Under the assumption that the transverse temperature is independent of the longitudinal velocity (see Sec. II B), the observed decrease in these measurements is modeled. The solid curves shown in Fig. 9 are the result of a simulation for transverse velocity distributions with different widths. The observed decrease is best described by a transverse velocity distribution with a width of around $5 \mathrm{~m} / \mathrm{s}$ (FWHM) in good agreement with the 3D Monte Carlo simulations. The measurements are very sensitive to the average velocity of the molecular beam that is used for correction. The transverse velocity distribution that is found can therefore be considered as a consistency check only. However, the fact that the measured points follow the calculated curves (irrespectively of the average velocity that is taken) indicates that the transverse velocity distribution is indeed independent of the final velocity of the decelerated beam.

From the simulations the emittance of the decelerated beam is found to be $[1.1 \mathrm{~mm} \times 6.5 \mathrm{~m} / \mathrm{s}] \times[1 \mathrm{~mm}$ $\times 5 \mathrm{~m} / \mathrm{s}]^{2}$, where the position and velocity spread indicate the FWHM of the fitted Gaussian distributions. These velocity distributions correspond to a translational temperature of $18 \mathrm{mK}$ and $11 \mathrm{mK}$ in the longitudinal and transverse direction, respectively. The ion signal is proportional to the density at the center of the trap. Typically, 125 ions/pulse are detected for the decelerated beam with a velocity of 91.8 $\mathrm{m} / \mathrm{s}$. As both the strength of the transition used for detection, and the exact size of the laser focus is not known, it is not possible to calculate a reliable number for the absolute density. However, a rough estimate can be made. The diffraction-limited waist of the laser focus is $35 \mu \mathrm{m}$. Using $10 \mathrm{~mJ} /$ pulse of laser radiation the transition is seen to saturate. It is assumed that all molecules within a cylinder with a diameter of two times the waist of the laser focus and a length of $2 \mathrm{~mm}$ (determined by the holes in the end cap of the trap) are ionized. Assuming a unit ion detection efficiency, a density of $2 \times 10^{7}$ molecules $/ \mathrm{cm}^{3}$ results. This is the density $24.5 \mathrm{~mm}$ behind the decelerator, the density just behind the decelerator is approximately five times higher. The total number of molecules in the decelerated bunch is $10^{5}$. These numbers can be assumed to be a lower limit. By combining the density in the beam with the emittance of the beam, the phase-space density is found. The definition for the phase-space density used here is $n_{0} \Lambda^{3}$ where $\Lambda$ is the thermal de Broglie wavelength, $\Lambda=\sqrt{2 \pi \hbar^{2} / m k T}$ and $n_{0}$ is the peak density [50]. The phase-space density for the decelerated beam is then found to be $4 \times 10^{-12}$. Similarly, the phase-space density of the ammonia molecules in $|J, M K\rangle$ $=|1,-1\rangle$ state in the source region of the beam is calculated to be $2 \times 10^{-11}$. This is the phase-space density at the peak velocity of the beam, the space density at the velocity of $271.5 \mathrm{~m} / \mathrm{s}$ selected by the decelerator, will be slightly lower. Therefore, the phase-space density in the decelerator decreases by about a factor of 4 , attributed to the fact that the hexapole has not been used during these experiments.

\section{SIMULTANEOUS DECELERATION OF AMMONIA ISOTOPOMERS}

The deceleration process as employed here depends on the Stark shift that the molecules experience in the applied electric fields. In the model used to describe this process, as presented in Sec. II A, the dependence on the exact shape of the electric field along the molecular beam axis is removed, and the motion is determined only by the difference in Stark energy before and after switching of the field. One can wonder, therefore, if it would be possible to simultaneously decelerate bunches of molecules having different masses and/or different Stark shifts. Let us consider the situation where molecules with a mass $m$ and experiencing a Stark shift $W(z)$ are decelerated using a time sequence for a phase angle $\phi_{0}$. The question now is whether for molecules with a different mass $m^{\prime}$ and/or a different Stark shift $W^{\prime}(z)$ a synchronous molecule exists for this time sequence, i.e., whether a molecule with a phase angle $\phi_{0}^{\prime}$ that is constant throughout the decelerator exists. If this synchronous molecule with mass $m^{\prime}$ exists, it will, by definition, traverse a distance $L$ in the time interval between subsequent switching times of the time sequence, just as the synchronous molecule with mass $m$ does. Therefore, the synchronous molecules with mass $m$ and $m^{\prime}$ will have the same average velocity and the same change in average velocity per deceleration stage. This implies that

$$
\frac{\Delta K^{\prime}\left(\phi_{0}^{\prime}\right)}{m^{\prime}}=\frac{\Delta K\left(\phi_{0}\right)}{m} .
$$

A synchronous molecule with mass $m^{\prime}$ can therefore be found, provided that $\left[\Delta K^{\prime}\left(\phi_{0}^{\prime}\right)\right]_{\text {max }} \geqslant\left(m^{\prime} / m\right) \Delta K\left(\phi_{0}\right)$, which is always fulfilled when the time sequence used is calculated for the molecule that can be least well decelerated. 


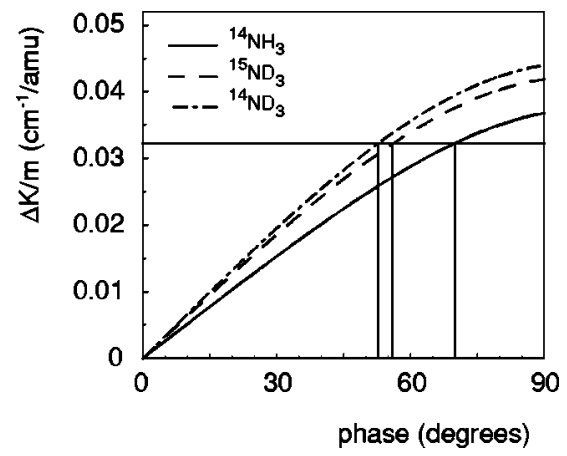

FIG. 10. The kinetic energy lost per stage divided by the mass for ${ }^{14} \mathrm{NH}_{3},{ }^{15} \mathrm{ND}_{3}$, and ${ }^{14} \mathrm{ND}_{3}$ molecules in the $|J, M K\rangle=|1,-1\rangle$ state, as a function of the phase angle $\phi_{0}$. The horizontal line shows $\Delta K\left(\phi_{0}\right) / m=0.032 \mathrm{~cm}^{-1} / \mathrm{amu}$.

Obviously, molecules with different masses and/or different Stark shifts will have different values of the phase angle $\phi_{0}$ and will thus have a different acceptance.

In Fig. $10, \Delta K\left(\phi_{0}\right) / m$ is shown as a function of the phase angle for ${ }^{14} \mathrm{NH}_{3},{ }^{15} \mathrm{ND}_{3}$, and ${ }^{14} \mathrm{ND}_{3}$ molecules. The horizontal line shows $\Delta K\left(\phi_{0}\right) / m=0.032 \mathrm{~cm}^{-1} / \mathrm{amu}$ corresponding to a phase angle of $\phi_{0}=70^{\circ}, 56^{\circ}$, and $53^{\circ}$ for ${ }^{14} \mathrm{NH}_{3},{ }^{15} \mathrm{ND}_{3}$, and ${ }^{14} \mathrm{ND}_{3}$, respectively.

In Fig. 11, TOF profiles recorded $24.5 \mathrm{~mm}$ behind the decelerator as a function of time after the start of the time sequence are shown for three different isotopomers of am-

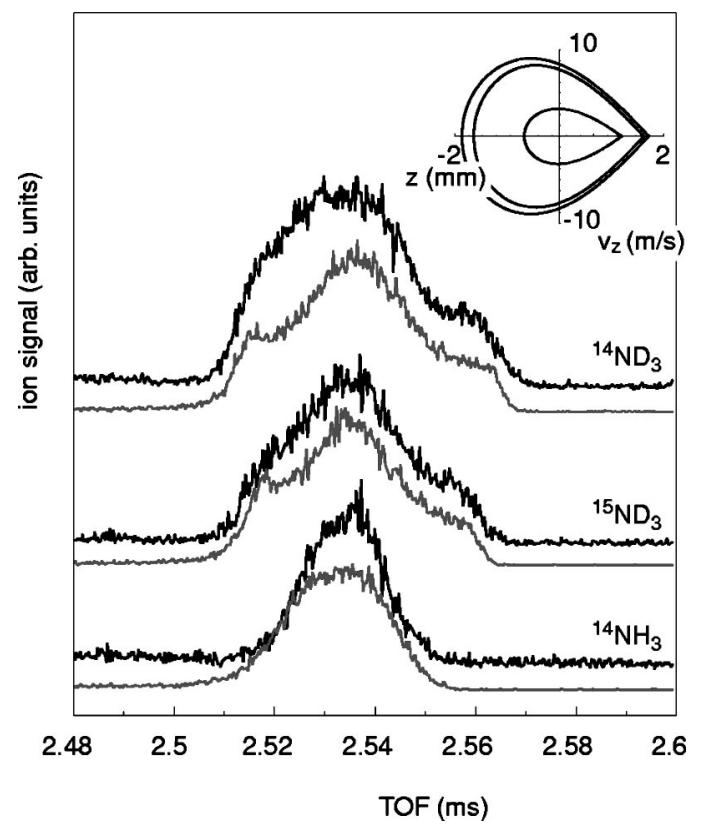

FIG. 11. Time-of-flight profiles for ${ }^{14} \mathrm{NH}_{3},{ }^{15} \mathrm{ND}_{3}$, and ${ }^{14} \mathrm{ND}_{3}$ molecules recorded $24.5 \mathrm{~mm}$ behind the decelerator as a function of time after the start of the time sequence. All TOF profiles are recorded using the time sequence calculated for ${ }^{14} \mathrm{NH}_{3}$ at a phase angle $\phi_{0}=70^{\circ}$. The gray curves show the results of 3D Monte Carlo simulations of the deceleration process. The area in phase space at the exit of the decelerator is indicated in the inset for ${ }^{14} \mathrm{NH}_{3}$ (inner curve), ${ }^{15} \mathrm{ND}_{3}$ (middle curve), and ${ }^{14} \mathrm{ND}_{3}$ (outer curve). monia. The measurements are performed using a time sequence that has been calculated for ${ }^{14} \mathrm{NH}_{3}$ at a phase angle $\phi_{0}=70^{\circ}$. The various ammonia isotopomers are decelerated from $256 \mathrm{~m} / \mathrm{s}$ to $96 \mathrm{~m} / \mathrm{s}$. It is evident from the measurements that the time integrated signal is larger for ${ }^{14} \mathrm{ND}_{3}$ and ${ }^{15} \mathrm{ND}_{3}$ than for ${ }^{14} \mathrm{NH}_{3}$. This is the combined effect of a larger spatial extent together with a larger longitudinal velocity spread of the decelerated beam, as indicated in the phase-space diagram in the inset. The small $(\sim 0.5 \mathrm{~mm})$ shift in the average position of the decelerated beam as seen in the phase-space diagram results in a small $(\sim 5 \mu \mathrm{s})$ increase in the overall time of flight for the deuterated ammonia molecules. The peculiar TOF profile observed for ${ }^{14} \mathrm{ND}_{3}$ and ${ }^{15} \mathrm{ND}_{3}$ reflects the phase-angle dependence of the transverse acceptance. Molecules contributing to the signal in the wings of the TOF profile have experienced large excursions in phase angle while traversing the decelerator. These molecules have, therefore, experienced widely varying transverse focusing forces (see Fig. 4). As explained in Sec. II B, this leads to a reduced transverse acceptance for these molecules. The results of 3D Monte Carlo simulations, shown underneath the experimental TOF profiles, are seen to reproduce the observations. The TOF profile for ${ }^{14} \mathrm{NH}_{3}$ is more symmetric, and effects due to transverse focusing are almost absent; the observed TOF profile can be reproduced equally well in a $1 D$ Monte Carlo simulation.

Experiments have been performed on other ammonia isotopomers like ${ }^{14} \mathrm{NHD}_{2}$ and ${ }^{14} \mathrm{NDH}_{2}$ as well. For these isotopomers the molecular symmetry is reduced, allowing these molecules to cool down to the absolute ground state. No low-field-seeking states were found to be populated in the beam.

\section{ELECTROSTATIC TRAP}

Electrostatic trapping of atoms and molecules was first considered by Wing [51]. The trap used here is very similar to the trap proposed in that original work. A cut through the trap along the $z$ axis is shown in Fig. 12. The geometry of the trap is the same as that of a Paul trap for charged particles [52]. However, instead of using rf fields as used for trapping charged particles, static electric fields are used for trapping neutral molecules. The trap consists of two end caps and a ring electrode. The inner radius $R$ of the ring electrode is 5 $\mathrm{mm}$. The end cap half-spacing is $R / \sqrt{2}$. In the end caps 2 $\mathrm{mm}$ diameter holes are made to enable the molecular beam to pass through. In the ring electrode $2 \mathrm{~mm}$ diameter holes are made to allow for laser detection of the trapped molecules at the center of the trap. The electric field in the trap has a quadrupole symmetry and is given by

$$
E=V\left(x^{2}+y^{2}+4 z^{2}\right)^{1 / 2} / R^{2},
$$

with $V$ the voltage difference applied between the ring electrode and the end caps and with the $(x, y, z)$ coordinates relative to the center of the trap. The absolute value of the electric field in the center is zero, and larger elsewhere. In weak electric fields the Stark shift of ${ }^{14} \mathrm{ND}_{3}$ and ${ }^{15} \mathrm{ND}_{3}$ is qua- 

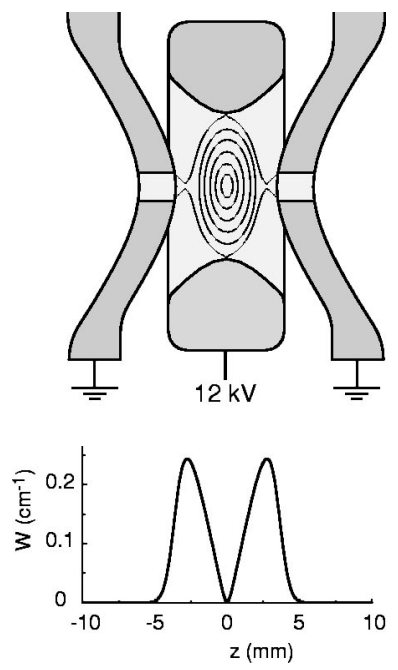

FIG. 12. Configuration of the electrostatic trap with the voltages as applied during trapping. In the trap, lines of equal electric field strength are shown. The lower curve shows the potential energy along the molecular beam axis ( $z$ axis) for ${ }^{14} \mathrm{ND}_{3}$ molecules in the $|J K\rangle=|11\rangle$ low-field-seeking state.

dratic and the restoring force close to the center is linear in the displacement. In higher electric fields the Stark shift becomes linear, and the force becomes constant. With a voltage difference of $12 \mathrm{kV}$ between the ring electrode and the end caps the maximum electric field of a closed contour of equal electric field is approximately $20 \mathrm{kV} / \mathrm{cm}$. The maximum electric field that occurs on the electrodes, which limits the voltage difference that can be applied, is about three times as high. For $\mathrm{ND}_{3}$ the trap depth is about $0.24 \mathrm{~cm}^{-1}$, or 350 $\mathrm{mK}$, as can be seen in the lower curve of Fig. 12. Near the center of the trap the motion is harmonic with an oscillation frequency $\Omega_{z} / 2 \pi=2 \Omega_{x} / 2 \pi=2 \Omega_{y} / 2 \pi=2 \mathrm{kHz}$.

The maximum velocity of ${ }^{14} \mathrm{ND}_{3}$ molecules, which can be confined in the trap with these settings, is approximately $17 \mathrm{~m} / \mathrm{s}$. In Fig. 13 lines of equal energy for ${ }^{14} \mathrm{ND}_{3}$ molecules are shown in phase space, representing the acceptance of the trap along the molecular beam direction. The phase-space diagrams of the trap in the $x$ and $y$ direction are the same as that in the $z$ direction, but with a two-times larger spatial acceptance in $x$ and $y$. In order to load the molecules into the trap without loss in phase-space density, the emittance of the

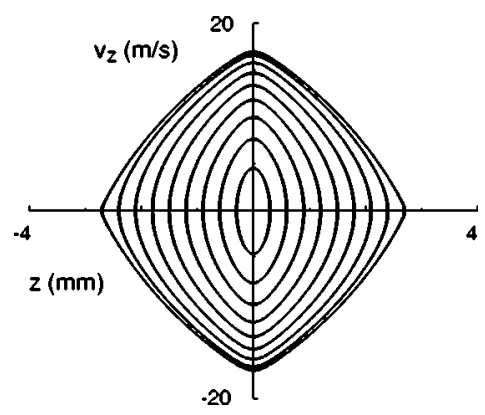

FIG. 13. Phase-space diagram for ${ }^{14} \mathrm{ND}_{3}$ molecules in the $|J K\rangle=|11\rangle$ low-field-seeking state. Lines of equal energy, representing the acceptance of the trap along the $z$ axis, are shown.

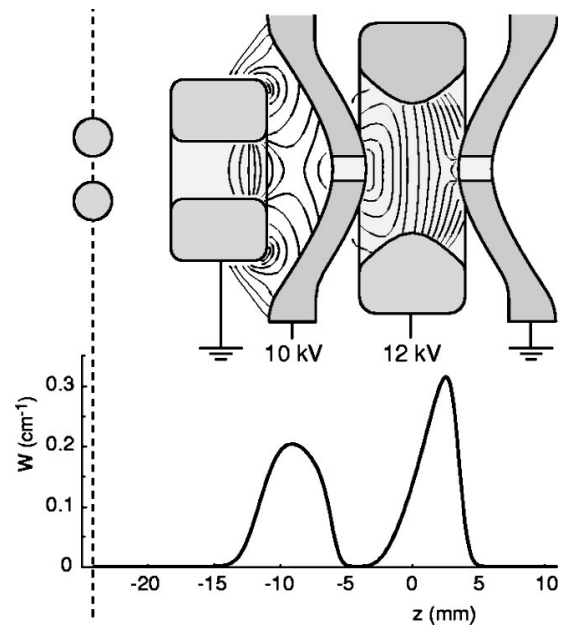

FIG. 14. A cut through the trap with the ring electrode, with voltages as applied for bunching and loading of the trap. The solid curves are lines of equal electric field. In the lower part of the figure the potential energy along the molecular beam axis for ${ }^{14} \mathrm{ND}_{3}$ in the $|J, K\rangle=|1,1\rangle$ low-field-seeking state is shown.

beam must be matched to the acceptance of the trap. This is done in two steps. First, the molecules are focused both in the transverse and longitudinal directions onto the entrance of the trap. Second, the molecules are decelerated upon entering the trap by applying the voltages asymmetrically to the trap electrodes. The average velocity of the beam is thereby reduced to zero at the center of the trap.

By using a phase angle of $\phi_{0}=70^{\circ}$ to decelerate ${ }^{14} \mathrm{ND}_{3}$ molecules, the emittance of the beam exiting the decelerator is $[1.1 \mathrm{~mm} \times 6.5 \mathrm{~m} / \mathrm{s}] \times[1 \mathrm{~mm} \times 5 \mathrm{~m} / \mathrm{s}]^{2}$. The absolute velocity spread of the beam remains constant throughout the decelerator. Therefore, by decelerating the beam the relative velocity spread and the divergence of the beam increase. To load the trap, the beam is decelerated down to $15 \mathrm{~m} / \mathrm{s}$. Over the $24.5 \mathrm{~mm}$ flight path from the decelerator to the trap, the density will decrease by more than two orders of magnitude. In order to focus the beam both in the longitudinal and transverse directions, a ring electrode with an inner diameter of 4 $\mathrm{mm}$ is mounted in front of the entrance end cap of the trap. By applying a voltage difference between this ring electrode and the entrance end cap, an electric field is generated with a minimum on the molecular beam axis, focusing molecules in low-field-seeking states in the transverse direction. Unlike in a hexapole, this minimum electric field will be nonzero. This nonzero field on the molecular beam axis can be used to focus the molecules in the longitudinal direction ("bunching").

In Fig. 14 a cut through the trap with the ring electrode in front is shown. Contours of equal electric field are drawn for an applied voltage difference of $10 \mathrm{kV}$ between the ring electrode and the entrance end cap of the trap. In the lower part of Fig. 14 the potential energy of ${ }^{14} \mathrm{ND}_{3}$ molecules in the $|J, K\rangle=|1,1\rangle$ low-field-seeking state along the molecular beam axis is shown. For the transverse and longitudinal focusing of the beam, the electric field distribution near the exit of the ring electrode, particularly the rising edge of the field in the $z=-16 \mathrm{~mm}$ to $-11 \mathrm{~mm}$ region, is important. In this 
region the potential energy along the molecular beam axis is nearly quadratically dependent on $z$. The molecules will experience a linear force $F=-k\left(z-z_{0}\right)$ with $z_{0}$ the position where the quadratic potential starts $\left(z_{0}=-16 \mathrm{~mm}\right)$ and $k$ the force constant. This force can be written in terms of the position $z_{s}$ of the synchronous molecule as $F=-k\left(z-z_{s}\right)$ $-k\left(z_{s}-z_{0}\right)$. The force is thereby separated in a restoring force towards the synchronous molecules and a force that lowers the velocity of the synchronous molecule. The restoring force leads to a rotation of the longitudinal phase-space distribution of the beam around the position of the synchronous molecule in phase space. In order for the molecules to rotate over the same angle in phase space, the potential must be switched on (and off) when all molecules are in the quadratic part of the potential. In our case, the range over which the potential is quadratic allows only the central part of the beam to be focused. When the potential is on during the appropriate time interval, this central part of the beam will refocus in free flight from the buncher to the trap, forming an image of the beam exiting the decelerator onto the entrance of the trap. The magnification of the image is determined by the ratio of the time of flight from the decelerator to the ring electrode to the time of flight from the ring electrode to the trap.

\section{TRAP-LOADING EXPERIMENTS}

In the upper part of Fig. 15, the TOF profiles for ${ }^{14} \mathrm{ND}_{3}$ molecules recorded in the trap are shown for four different times, indicated by arrows, at which the electric field of the buncher is switched off. The time intervals $\Delta T$ given in the figure are the intervals between the time at which the synchronous molecule exits the decelerator $(2.830 \mathrm{~ms}$ after start of the time sequence) and the times at which the buncher is switched off. The lower curve shows the TOF profile when the buncher is not used. These measurements reflect the spatial distribution of the beam inside the trap, which is considerably narrowed by using the buncher. When the electric field of the buncher is switched off at later times, the molecules are decelerated more and are seen to arrive later at the center of the trap. In addition, the signal is increased due to transverse focusing of the beam, which is optimal at later times. The gray curves underneath the measurements show the results of 3D Monte Carlo simulations of the experiment. The simulations are seen to qualitatively reproduce both the longitudinal and transverse focusing of the beam. Since the outer shape of the entrance end cap electrode, and thus the electric field in front of the electrostatic trap, is rather poorly defined in our case, a better agreement would not be expected. In the lower part of Fig. 15, the evolution of the beam in phase space is shown for the situation where the buncher is switched off $800 \mu$ s after the synchronous molecule exits the decelerator. In this case the central part of the beam is spatially bunched at the entrance of the trap. The bunched part of the beam occupies a phase-space area of [2 $\mathrm{mm} \times 4 \mathrm{~m} / \mathrm{s}$ ] in the forward direction, and has an average velocity of $12 \mathrm{~m} / \mathrm{s}$. In the transverse direction the image is less well-defined and is in practice determined by the size of the hole in the entrance end cap of the trap.
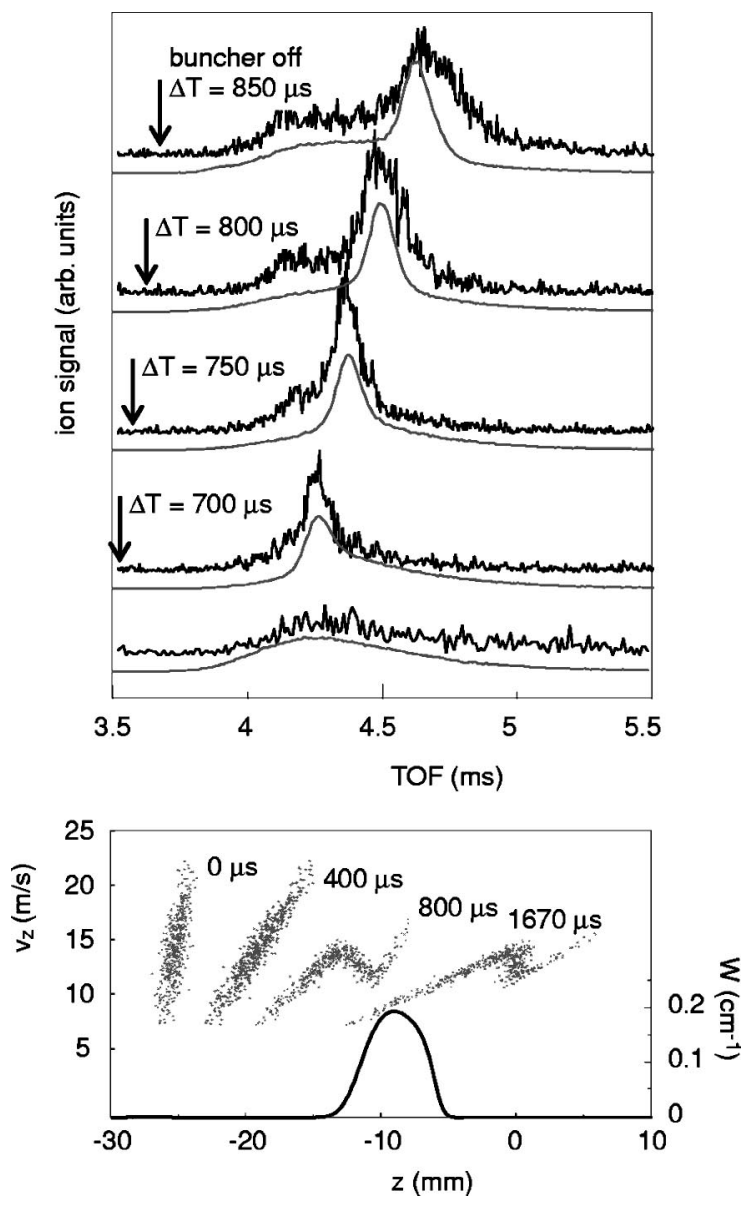

FIG. 15. Time-of-flight profiles for ${ }^{14} \mathrm{ND}_{3}$ molecules recorded at the center of the trap for four different times, indicated by arrows, at which the buncher is switched off. The time intervals $\Delta T$ between the time at which the synchronous molecule exits the decelerator (2.830 $\mathrm{ms}$ on the horizontal axis) and the times at which the buncher is switched off, are given. The lower curve shows the timeof-flight profile of the beam when the buncher is not used. The gray curves show the results of 3D Monte Carlo simulations of the experiment. In the lower part of the figure the phase-space diagrams of the beam are shown for different times after exiting the decelerator, together with the potential energy $W(z)$ (right axis) along the molecular beam axis for ${ }^{14} \mathrm{ND}_{3}$ in the $|J, K\rangle=|1,1\rangle$ low-fieldseeking state.

In order to bring the average velocity of the beam down to zero, the voltages to the trap electrodes are applied asymmetrically, as shown in Fig. 14. With a voltage of $10 \mathrm{kV}$ on the entrance end cap and $12 \mathrm{kV}$ on the ring electrode, the electric field will be small at the entrance of the trap and will increase towards the center of the trap. The potential energy of ${ }^{14} \mathrm{ND}_{3}$ molecules in the $|J K\rangle=|11\rangle$ low-field-seeking state along the molecular beam axis is shown in the lower part of Fig. 14. The synchronous molecule entering the trap with a velocity of $12 \mathrm{~m} / \mathrm{s}\left(E_{k i n}=0.12 \mathrm{~cm}^{-1}\right)$ will come to a standstill at the center of the trap. The potential energy is nearly quadratically dependent on the position along the molecular beam axis. Therefore, in the time that the synchronous molecule is slowed down to zero the whole beam is rotated over an angle of $\frac{1}{2} \pi$ in phase space. This is identical 

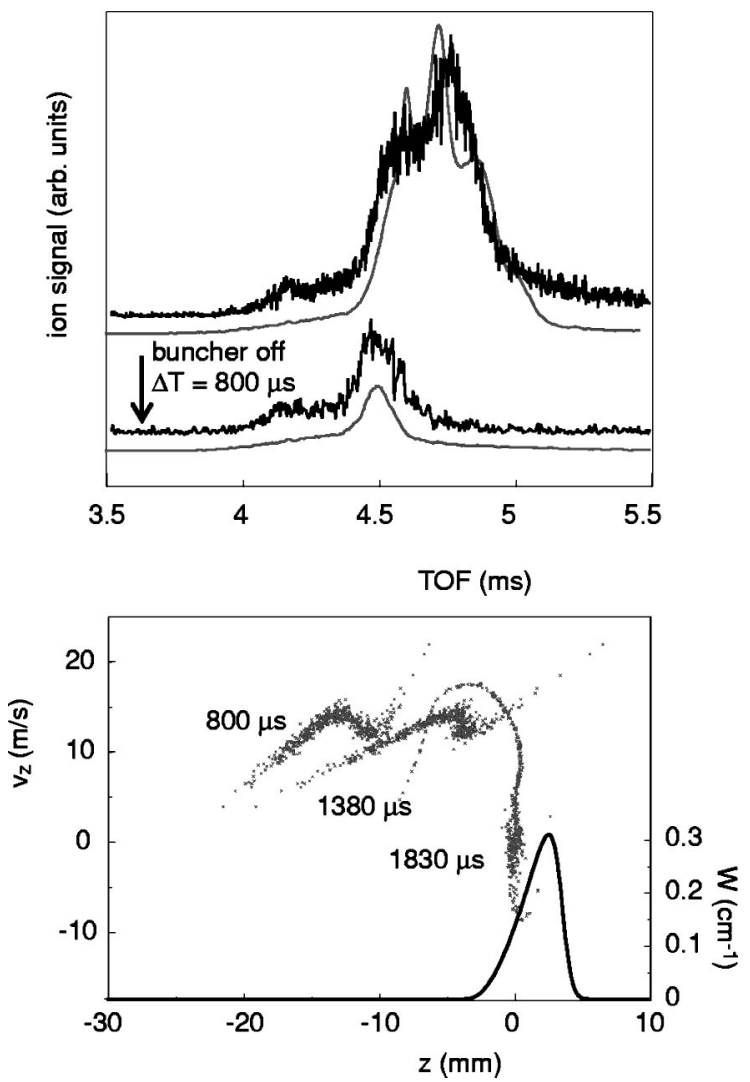

FIG. 16. Time-of-flight profiles for ${ }^{14} \mathrm{ND}_{3}$ molecules recorded at the center of the trap with the buncher switched off at $\Delta T$ $=800 \mu \mathrm{s}$. The upper curve is recorded with voltages applied asymmetrically to the trap electrodes. The lower curve is recorded without deceleration of the molecules upon entering the trap. The gray curves show the results of 3D Monte Carlo simulations of the experiment. In the lower part of the figure the phase-space diagrams of the beam are shown, together with the potential energy $W(z)$ (right axis) along the molecular beam axis for ${ }^{14} \mathrm{ND}_{3}$ in the $|J, K\rangle$ $=|1,1\rangle$ low-field-seeking state.

to keeping the original phase-space distribution and simply interchanging the velocity and position axes, with the appropriate scaling.

In Fig. 16 the TOF profiles for ${ }^{14} \mathrm{ND}_{3}$ molecules are recorded at the center of the trap with the buncher switched off $800 \mu$ s after the synchronous molecule has exited the decelerator. The lower curve is recorded without deceleration of the molecules inside the trap, and is the same as the curve shown in Fig. 15. In the upper curve, the molecules are decelerated upon entering the trap. When the voltages on the trap electrodes are kept on, the trap will act as a concave mirror for the ammonia molecules. The upper curve shows the density of ammonia molecules at the center of the trap while the molecules are reflected from the electrostatic mirror. The increased signal of the upper curve relative to the lower one is due to bunching and transverse focusing when the electric field is on. The gray curves show the results of 3D Monte Carlo simulations of the experiment. In the lower part of Fig. 16 the phase-space diagrams of the beam are shown for different times after exiting the decelerator, together with the potential energy $W(z)$ (right axis) along the

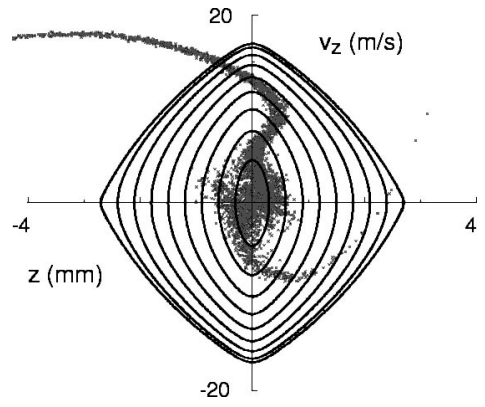

FIG. 17. The phase-space distribution of the beam at $\Delta T$ $=1830 \mu \mathrm{s}$ at the position of the trap together with the acceptance diagram of the trap.

molecular beam axis for ${ }^{14} \mathrm{ND}_{3}$ in the $|J, K\rangle=|1,1\rangle$ lowfield-seeking state. The phase-space diagram at $1.830 \mathrm{~ms}$ shows the beam when the synchronous molecule, entering the trap with $12 \mathrm{~m} / \mathrm{s}$, has come to a standstill. The central part of the beam occupies a phase-space area of $[0.8 \mathrm{~mm}$ $\cdot 10 \mathrm{~m} / \mathrm{s}]$ in the forward direction. This phase-space distribution is shown enlarged in Fig. 17, with the longitudinal acceptance diagram of the trap as an overlay.

\section{TRAPPING EXPERIMENTS}

In order to trap the molecules the voltage on the entrance end cap is switched off once the synchronous molecule has come to a standstill. In Fig. 18 the density of ${ }^{14} \mathrm{ND}_{3}$ molecules at the center of the trap is shown as a function of time. The trap is switched on $1.830 \mathrm{~ms}$ after the synchronous molecule exits the decelerator, i.e., $4.660 \mathrm{~ms}$ after the start of the time sequence. Shortly after the trap is switched on the density at the center of the trap is seen to oscillate. These oscillations have faded away after several milliseconds and a steady signal from the trapped ammonia molecules remains. The inset in the figure shows the ammonia density on a longer time scale. The ammonia signal intensity is seen to exponentially decay with a $1 / e$ time of $0.3 \mathrm{~s}$, although at

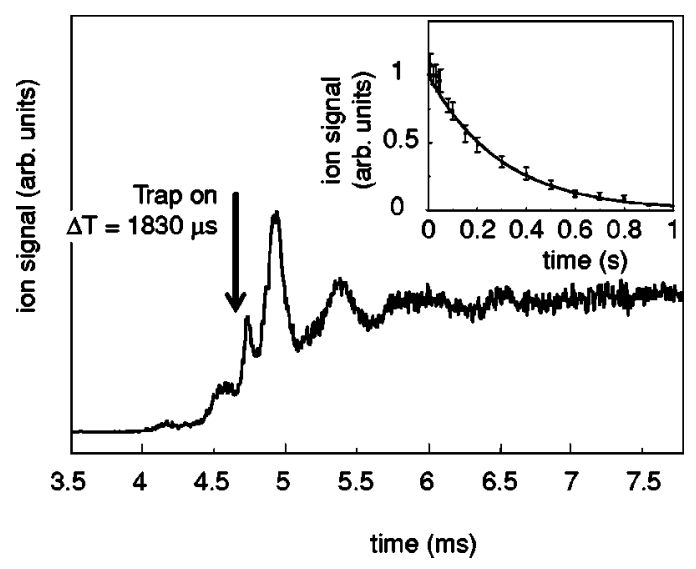

FIG. 18. Density of ${ }^{14} \mathrm{ND}_{3}$ molecules recorded at the center of the trap as a function of time. The time at which the trap is switched on is indicated with an arrow. The inset shows the density of ${ }^{14} \mathrm{ND}_{3}$ molecules on a longer time scale, and an exponential fit to the data (solid line) with a $1 / e$ decay time of $0.3 \mathrm{~s}$. 
short times the decay appears to be slightly faster. The observed trap loss is attributed to collisions with background gas in the vacuum chamber; the background pressure of typically $2 \times 10^{-8}$ Torr is consistent with the observed trap loss rate.

The oscillations that are observed shortly after the trap is switched on, indicate that the emittance of the beam is not perfectly matched to the acceptance of the trap. Two situations can be distinguished: (i) the emittance and the acceptance are not centered around the same point in phase space, i.e., the average position of the beam is not at the center of the trap and/or the average velocity of the beam is nonzero when the trap is switched on, and (ii) the emittance of the beam and the acceptance of the trap do not have the same shape in phase space, i.e., the ratio of the spatial distribution to the velocity distribution of the beam is different from that of the acceptance diagram of the trap. These two situations lead to different types of oscillations. In the first situation, the whole beam will oscillate back and forth in the trap. In the second situation, the beam will perform a breathing motion in the trap. The oscillations will decay due to the anharmonicity of the trap potential.

In order to experimentally study these oscillations, the density of ${ }^{14} \mathrm{ND}_{3}$ molecules is measured at two positions on the molecular beam axis, symmetrically located around the center of the trap. In the observed oscillation pattern at these two positions, the two types of oscillation will appear in a different manner. The oscillation of the whole beam back and forth in the trap results in an oscillation at the longitudinal trap frequency of $2 \mathrm{kHz}$; the measurements at the two positions will have a phase difference of $180^{\circ}$. The breathing motion in the trap results in an oscillation at twice the longitudinal trap frequency, with the same phase at both locations.

In Fig. 19 the density of ${ }^{14} \mathrm{ND}_{3}$ molecules in the trap is shown as a function of time. Pairs of measurements are taken under identical experimental conditions but with the detection laser focused at $z=+0.35 \mathrm{~mm}$ and $z=-0.35 \mathrm{~mm}$. The middle set of measurements are recorded when the trap is switched on at $1.830 \mathrm{~ms}$ after the synchronous molecule has exited the decelerator. This is the time at which, according to the calculations, the synchronous molecule has come to a standstill at the center of the trap (see Fig. 17). The upper and lower measurements are recorded when the trap is switched on $80 \mu$ s later and earlier, respectively. Although oscillations can be recognized in all three sets of measurements, it is evident that these oscillations are least pronounced when the trap is switched on at $1.830 \mathrm{~ms}$, which thus experimentally appears to be the right time indeed. In this case the signal intensity away from the center of the trap is slightly less than in the other measurements, as expected. Under these conditions, the signal intensity at the center of the trap, shown in Fig. 18, is optimal. When the trap is switched on too late, as for the upper curves, the molecules are already reflected by the mirror and the signal intensity is minimum (maximum) downstream (upstream) from the center of the trap. In the upper curve taken at $z=+0.35 \mathrm{~mm}$, the oscillation back and forth in the trap with a period of approximately $0.5 \mathrm{~ms}$ is clearly visible. Although still vis-

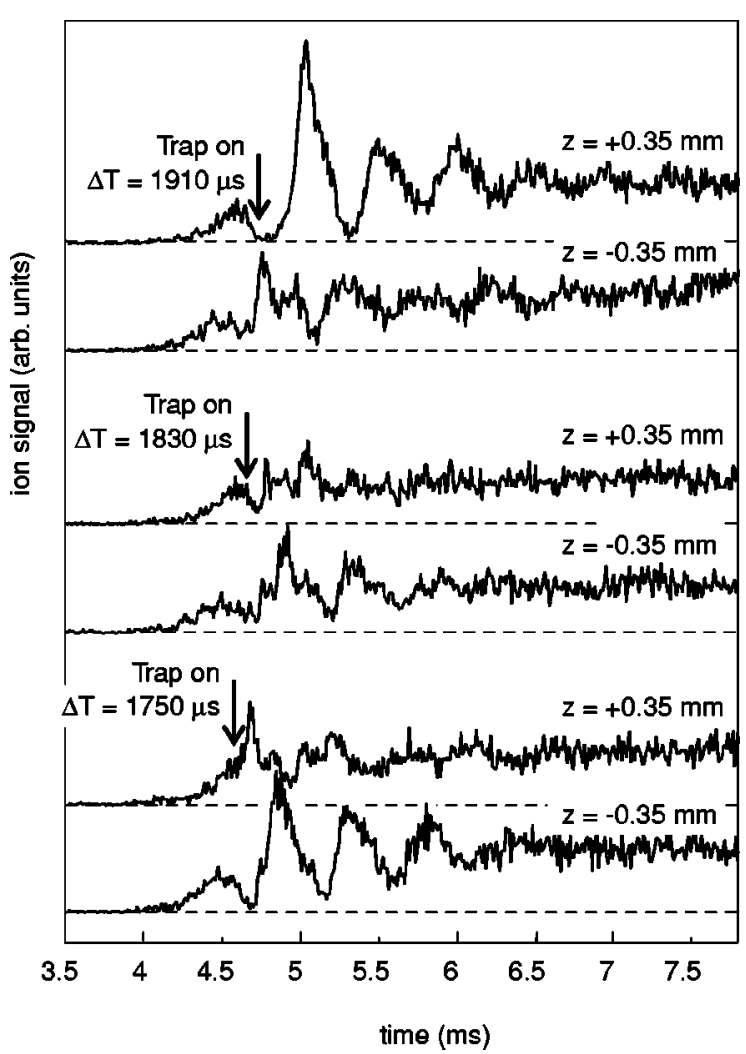

FIG. 19. Density of ${ }^{14} \mathrm{ND}_{3}$ molecules recorded in the trap as a function of time, at two different positions on the $z$ axis. The three sets of measurements show the dependence of the oscillation pattern on the time at which the trap is switched on.

ible, the oscillation back and forth is less pronounced in the curve recorded at $z=-0.35 \mathrm{~mm}$. This indicates that also a breathing motion is present, which can be recognized as such at twice the longitudinal trap frequency. The measurements that are taken when the trap is switched on $80 \mu$ s too early, are similar to the ones that are taken when the trap is switched on too late; the measurements taken at $z=$ $-0.35 \mathrm{~mm}(z=+0.35 \mathrm{~mm})$ when the trap is switched on too early have to be compared to the measurements taken at $z=+0.35 \mathrm{~mm}(z=-0.35 \mathrm{~mm})$ when the trap is switched on too late. In the measurements taken at $1.830 \mathrm{~ms}$ the oscillation back and forth in the trap is largely absent. The only oscillation expected to be present in this case is due to the breathing motion in the trap. Although frequencies around twice the trap frequency are observed in both curves, the curves are less similar than expected. This is probably caused by the anisotropic phase-space distribution of the beam when the trap is switched on, as seen in Fig. 17.

The transverse oscillations back and forth and the transverse breathing motion in the trap are expected at $1 \mathrm{kHz}$ and $2 \mathrm{kHz}$, respectively. In order to study these oscillations measurements need to be performed with the laser beam focused symmetrically above and below the molecular beam axis. Such measurements are more complicated in the current setup, as the detection efficiency is strongly position dependent off axis, and have not been performed. In the measurements shown in Fig. 19 it is not clear if transverse oscillations are present at all. However, it might be that the remaining $2 \mathrm{kHz}$ 
oscillation in the measurement shown in Fig. 18, taken at the center of the trap when the trap is switched on at the right time, is due to the transverse breathing motion in the trap.

The longitudinal and transverse distributions generally do not have the same temperature, even when the phase-space distribution of the decelerated beam perfectly matches the acceptance of the trap. In the absence of collisions, redistribution of molecules in the trap will lead to a change in temperature due to a coupling of the longitudinal and transverse motion. This can be an alternative explanation for the observed faster trap loss during the first $20 \mathrm{~ms}$ of trapping, as seen in Fig. 18.

In order to determine the temperature of the trapped ammonia molecules, the spatial distribution of the molecules in the trap is measured along the $z$ axis. This is done by scanning the position of the focused detection laser through the 2 $\mathrm{mm}$ diameter holes in the ring electrode of the trap. For this measurement the trap is switched on at $1.830 \mathrm{~ms}$. The detection laser is fired after the molecules have been trapped for $50 \mathrm{~ms}$, long compared to the oscillation periods in the trap. Since the density in the trap is low, no collisions between trapped molecules will take place during this time interval. Strictly speaking, temperature is not defined in this case; it is used here as a measure of the average energy of the molecules in the trap. Knowing the trapping potential for ${ }^{14} \mathrm{ND}_{3}$ molecules in the $|J, K\rangle=|1,1\rangle$ low-field-seeking state, the spatial and velocity distributions can be calculated assuming a certain temperature [50]. To make sure that ion detection is not disturbed by residual electric fields, the detection laser is actually fired $20 \mu$ s after the voltages on the trap electrodes are switched off. During this $20 \mu$ s time interval the molecular cloud will slightly expand, which is included in the simulations.

In Fig. 20 the density of trapped ${ }^{14} \mathrm{ND}_{3}$ molecules is shown as a function of the position along the $z$ axis. The detection efficiency is constant over the range in $z$ covered in these measurements. Each data point is averaged over ten trapping cycles. The error bar indicates the statistical spread in the measurements. The solid curves are the results of a Monte Carlo simulation, assuming a thermal distribution of molecules in the trapping potential. Detection of molecules in a $70-\mu \mathrm{m}$-diameter, 2 -mm-long cylinder is assumed. The measurements are best described using a thermal distribution with a temperature of $25 \mathrm{mK}$. In principle, similar measurements could be performed to determine the spatial distribution in the transverse direction as well. As discussed, these measurements are more complicated and have not been performed.

The maximum number of ammonia ions detected per laser pulse is approximately 45. Assuming a unit ionization efficiency of the ammonia molecules in a 2-mm-long, $70-\mu$ m-diameter volume of the laser focus and with a unit ion detection efficiency, a lower limit for the peak density of trapped ${ }^{14} \mathrm{ND}_{3}$ of $n_{0}=1.3 \times 10^{7}$ molecules $/ \mathrm{cm}^{3}$ results. The total number of molecules in the trap is then approximately $10^{4}$ - a factor of 10 less than the number of molecules that exited the decelerator. Together with the measured temperature of $25 \mathrm{mK}$, corresponding to a thermal de Broglie wavelength of $\Lambda=2.5 \mathrm{~nm}$, this yields a phase-space density $n_{0} \Lambda^{3}$

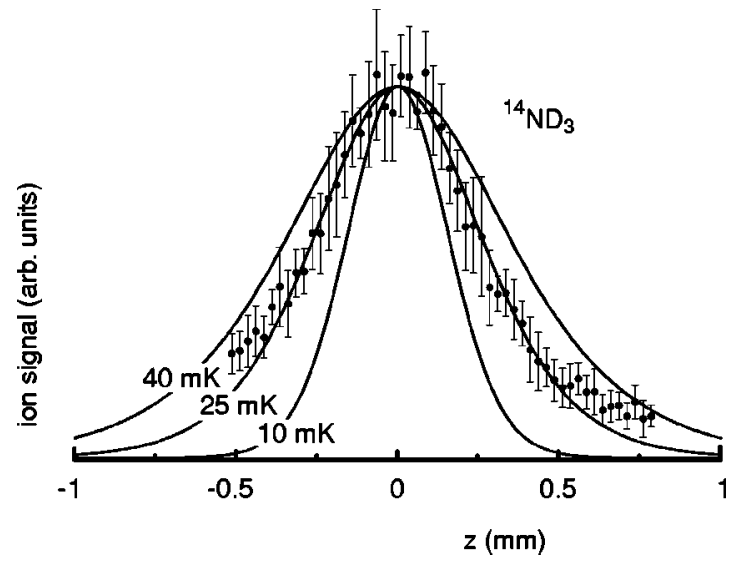

FIG. 20. Density of trapped ${ }^{14} \mathrm{ND}_{3}$ molecules recorded as a function of the position along the $z$ axis. The solid curves show the results of a Monte Carlo simulation for thermal distributions with different temperatures.

of $2 \times 10^{-13}$. This can be compared to the phase-space density of the original beam, which is calculated to be 2 $\times 10^{-11}$. For the trapping experiment the beam is decelerated starting from an initial velocity of $256 \mathrm{~m} / \mathrm{s}$, where the phasespace density is about a factor of 2 lower than at the peak velocity. The overall loss in phase-space density from the beam to the trap is therefore approximately 50 . As discussed earlier, there is a factor of 4 loss in phase-space density from the matching of the molecular beam onto the decelerator. The remaining factor of 12 loss results from the (nonideal) loading of the decelerated beam into the trap.

In Fig. 21 the ion signal resulting from the trapped sample of ${ }^{14} \mathrm{ND}_{3}$ molecules is shown together with the signal obtained at the center of the trap for ammonia molecules in the original molecular beam at the central velocity of $285 \mathrm{~m} / \mathrm{s}$, and in the decelerated beam at a velocity of $92 \mathrm{~m} / \mathrm{s}$.

Identical measurements have been performed on ${ }^{15} \mathrm{ND}_{3}$ using the same time sequence as in the experiment on ${ }^{14} \mathrm{ND}_{3}$. Although it has been detailed in Sec. V that the same time sequence can be used for deceleration of both the isotopomers, it is not a priori clear that this holds for bunching and trap loading as well. One might expect that in the present setup an optimum is found for ${ }^{15} \mathrm{ND}_{3}$ at different settings

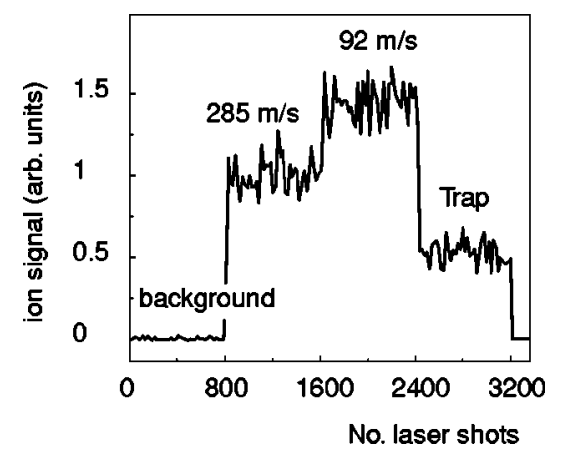

FIG. 21. The density of ${ }^{14} \mathrm{ND}_{3}$ molecules recorded at the center of the trap for the original molecular beam at the central velocity of $285 \mathrm{~m} / \mathrm{s}$, for the decelerated beam at a velocity of $92 \mathrm{~m} / \mathrm{s}$ and for the trapped molecules. Each data point is averaged over 20 laser shots. 


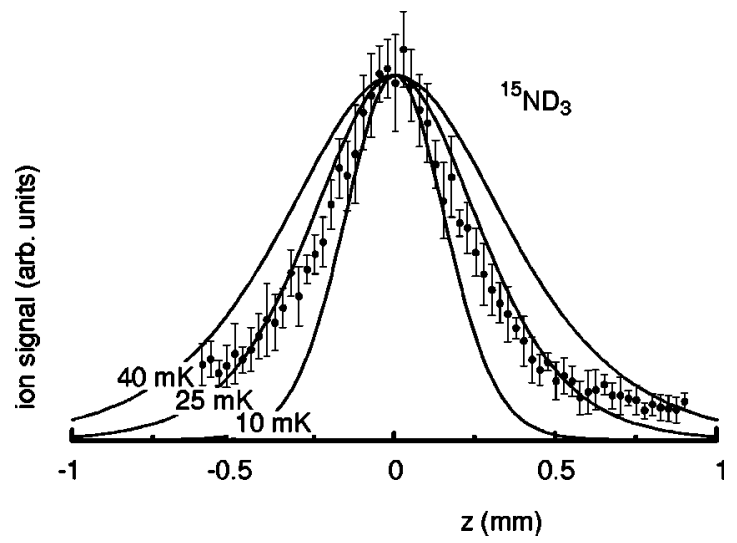

FIG. 22. Density of trapped ${ }^{15} \mathrm{ND}_{3}$ molecules as a function of the position along the $z$ axis. The solid curves show the results of a Monte Carlo simulation for thermal distributions with different temperatures.

than used for ${ }^{14} \mathrm{ND}_{3}$. This has not been investigated further. The measurements for the spatial extent of ${ }^{15} \mathrm{ND}_{3}$ in the trap is shown in Fig. 22. The measurements are well reproduced when a temperature of $25 \mathrm{mK}$ is assumed. Within the experimental accuracy the absolute signal is the same for both the isotopomers of ammonia. Both isotopomers have also been trapped simultaneously at a factor of 2 lower peak density for each species.

In Fig. 23, $(2+1)$-REMPI spectra of ${ }^{14} \mathrm{ND}_{3}$ are shown, recorded via intermediate states of different vibrational symmetry. The spectra are recorded under conditions close to saturation, and the width of the lines is determined by power broadening. All spectra are recorded on the same intensity scale, and are averaged over 40 shots. In the upper panel, the $\nu_{2}^{\prime}=4$ vibrational level in the $\widetilde{B}^{1} E^{\prime \prime}$ state is used as intermediate. Via this level, only molecules in the lower inversion doublet levels (vibrational $s$ symmetry) in the electronic and vibrational ground state are detected. Measurements are shown for the molecular beam (upper trace) and for trapped molecules (lower trace). The $J_{K}$ rotational quantum numbers of the ground-state levels are indicated in the figure [53]. In the lower panel, the $\nu_{2}^{\prime}=5$ vibrational level is used as intermediate, only allowing detection of molecules in the upper inversion doublet levels (vibrational $a$ symmetry).

In the beam, both $s$ and $a$ components of the $0_{0}, 1_{0}$, and $1_{1}$ levels are observed. The $1_{0}$ and $1_{1}$ level are located $10 \mathrm{~cm}^{-1}$ and $8 \mathrm{~cm}^{-1}$ above the rotational ground-state level, respectively. The $1_{1}$ level is the only para level that is populated in the beam, indicating a rotational temperature below $3 \mathrm{~K}$. The spectra recorded on the trapped molecules only show the five allowed transitions originating from the upper inversion doublet level of the $1_{1}$ state.

\section{CONCLUSIONS AND FUTURE PROSPECTS}

In this paper a method to decelerate and trap polar molecules using time varying electric fields is demonstrated. It is shown that the high phase-space density of molecules that is present in the moving frame of a pulsed molecular beam can, in principle, be transferred to the laboratory frame without
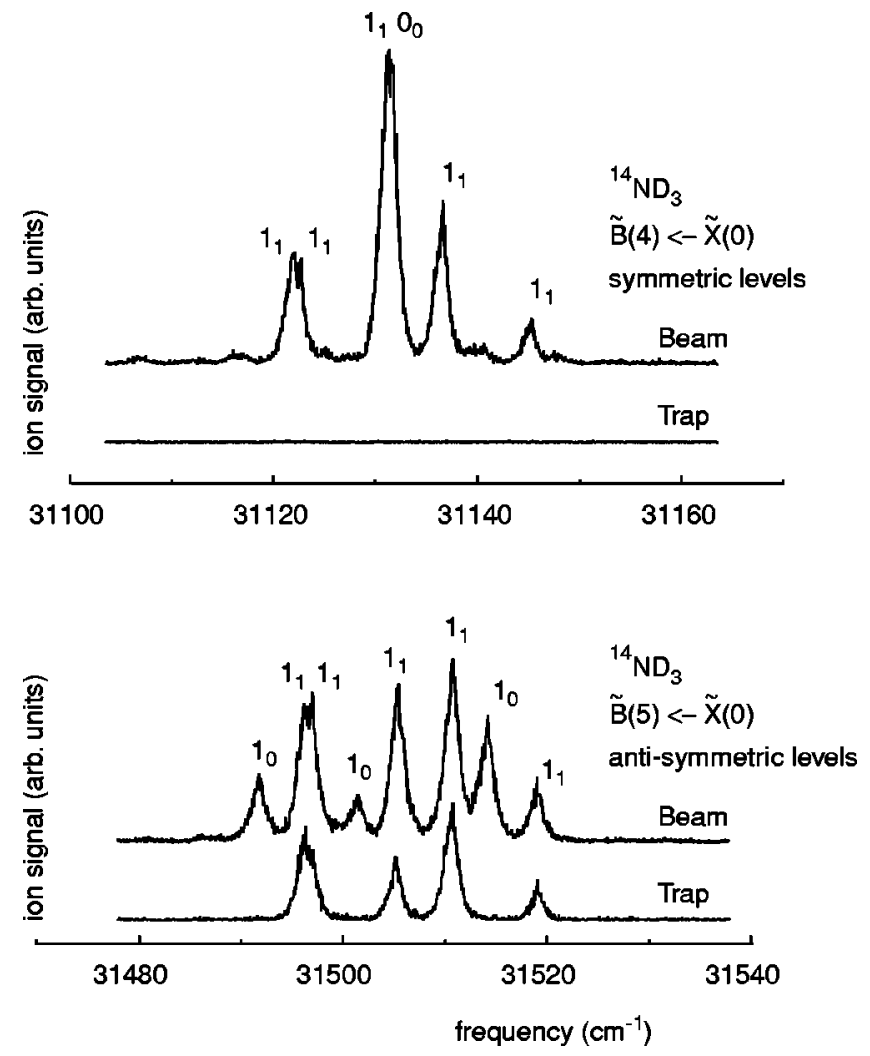

FIG. 23. (2+1)-REMPI spectra recorded via the $\widetilde{B}^{1} E^{\prime \prime}, \nu_{2}^{\prime}$ $=4$ (upper panel) and $\nu_{2}^{\prime}=5$ (lower panel) state in ${ }^{14} \mathrm{ND}_{3}$. In each panel, the upper curves are from measurements on the molecular beam, whereas the lower curves are recorded on the trapped molecules.

loss. Different molecules and isotopomers can be trapped simultaneously. On the order of $10^{4},{ }^{14} \mathrm{ND}_{3}$ molecules are trapped at a density of over $10^{7}$ molecules $/ \mathrm{cm}^{3}$ and at a temperature of $25 \mathrm{mK}$. The corresponding phase-space density in the trap is $2 \times 10^{-13}, 50$ times less than the initial phase-space density in the beam. This loss can be reduced with a better matching of the decelerated beam to the trap.

Ultimately, the phase-space density in the trap is limited by the phase-space density that can be obtained in the beam. In a pulsed supersonic expansion, densities of $10^{13}$ molecules $/ \mathrm{cm}^{3}$ at a translational temperature of $1 \mathrm{~K}$ are routinely obtained [45]. Phase-space densities on the order of $10^{-8}$ appear feasible. Compared to this, the measured phasespace density in our beam is rather low, apparently due to the small pumping capacity in the current setup. Furthermore, $\mathrm{Xe}$ is used as a carrier gas to start with a low velocity of the initial beam. Due to its large polarizability, Xe readily forms clusters and is therefore not considered to be the ideal carrier gas. The use of $\mathrm{Kr}$ or Ar as a carrier gas may lead to a more intense initial beam, requiring a longer decelerator, however.

To increase the number of trapped molecules the area in phase space that is imaged from the beam source to the trap should be increased. Currently, the area in phase space that can be imaged is limited by the buncher. By using a buncher consisting out of multiple ring electrodes and a hexapole focuser, this can be largely improved. The acceptance of the 
decelerator can be increased by using higher electric fields or by using a larger spacing between the electrodes, at the same value of the electric field. By using more deceleration stages, the decelerator can be operated at a lower phase angle. Optimization of the overall deceleration and trapping process will be performed via feedback control optimization of the time sequence, using genetic learning algorithms. For a given experimental geometry and molecule, this optimization needs to be performed only once.

Another possibility to increase the number of trapped molecules would be to accumulate molecules from successive deceleration cycles in the trap. An increase of phasespace density via this accumulation is only possible in specific cases, e.g., for the $\mathrm{NH}$ radical, where laser-induced spontaneous decay provides a unidirectional path to reload a trap [54]. It is always possible to increase the total number of molecules while keeping the phase-space density constant. This process is known as phase-space stacking in accelerator physics [49].

With the scheme outlined in this article, any polar molecule can be decelerated and trapped provided that it has a sufficiently large positive Stark shift in experimentally attainable electric fields. In Table I a selection of polar molecules suited for deceleration and trapping is given. The acceleration experienced by the molecules in the beam depends on the ratio of their (positive) Stark shift to their mass. This is given as a "figure of merit" in Table I, scaled relative to ${ }^{14} \mathrm{ND}_{3}$. Unless noted otherwise the Stark shift has been calculated at an electric field of $200 \mathrm{kV} / \mathrm{cm}$. The number of stages that would be required to bring the molecules to a standstill in the present setup, i.e., with electric fields on the axis of the decelerator of $90 \mathrm{kV} / \mathrm{cm}$, is given. For this, the molecules are assumed to have an initial velocity determined by the Xe carrier gas at a temperature determined by the vapor pressure of the molecules. For stable molecules, the temperature at which the vapor pressure is $0.1 \mathrm{~atm}$ is taken; for radicals a room-temperature expansion is assumed. Most of the molecules that have been listed have been used extensively in hexapole focusing experiments, in which case a reference to these experiments is given. Both the production (in a molecular beam) and the detection methods for the molecules listed in Table I are well known. For radicals such as $\mathrm{OH}$, production of intense pulsed beams with densities above $10^{11}$ molecules $/ \mathrm{cm}^{3}$ per quantum state have been reported [70].

The use of electrostatic fields to trap molecules, offers many possibilities. Electric fields can be made in a wide variety of shapes, and can be ramped and switched rapidly. Molecules can be trapped around a charged wire [71-74]. With a hexapole bent into a torus, a storage ring for polar molecules can be constructed [36,75]. ac traps can be constructed to trap molecules in high-field-seeking states $[76,77]$. It should be possible to produce microcircuits to manipulate and trap molecules on a chip. Electrostatic trapping fields can easily be superimposed with magnetic traps [54], optical traps [22], or high- $Q$ optical cavities [78].

Ultrahigh resolution spectroscopy will greatly benefit from the increased interaction time and the reduced thermal broadening offered by cold molecules. Molecules are used in the search for violation of time reversal symmetry [79] and in the study of weak interactions in chiral molecules [80]. By accurately measuring the ratio of the transition frequencies of various types of optical transitions in molecules, the temporal variation of the fine-structure constant might be determined in a laboratory experiment [81]. The use of cold molecules is expected to increase the precision of these studies by orders of magnitude.

The long storage time in traps makes it possible to study the lifetimes of vibrationally or electronically excited metastable states. Molecules can be prepared in these states either in the trap or prior to deceleration. Closed rovibrational transitions might actually be used to cool the trapped molecules further via laser cooling.

The study of ultracold collisions and reactions forms an interesting research topic. At low temperatures the associated de Broglie wavelength of the molecules becomes of the same size as the collision complexes, dramatically changing the interactions at these temperatures [2]. Besides studying collisions among trapped species, interesting effects are predicted for collisions at nonzero collision energies. Calculations show that elastic and inelastic cross-sections show sharp resonances at low collision energies, increasing by two orders of magnitude at these energies [82]. Measuring the width and position of these resonances can be performed by sending a velocity tuneable molecular beam through the sample of trapped molecules loaded from a previous cycle. Scattering resonances occur, for instance, when colliding molecules begin to rotate, leaving them with insufficient translational kinetic energy to overcome their van der Waals attraction. The formation of long-lived, transiently bound, molecular complexes might enable reactions to occur via tunneling through reaction barriers, opening up novel routes for low-temperature chemistry.

With a sufficiently high density of trapped molecules, the regime of evaporative cooling might be reached [83]. The success of evaporative cooling depends on the ratio of the elastic to inelastic collision rate of the trapped molecules. This ratio depends critically on molecular properties such as the magnitude of the dipole moment and of the zero-field splitting [84]. As the trapping scheme presented here can be used for a large number of different molecules, one can hope to find a suitable candidate molecule. Alternatively, polar molecules can be trapped in their lowest-energy quantum state in an ac trap, by which inelastic collisions can be avoided. In this case additional heating due to the rf fields will take place. It is expected, however, that under certain conditions evaporative cooling will dominate over this heating [85].

Another viable method to cool the molecules further might be sympathetic cooling with laser-cooled atoms. Recently, Bose-Einstein condensation of ${ }^{41} \mathrm{~K}$ atoms has been achieved via sympathetic cooling with a ${ }^{87} \mathrm{Rb}$ gas [86].

When further cooling of the trapped molecules proves to be feasible, collective effects can be studied in these samples. If a molecular Bose-Einstein condensate is formed, there is the possibility to study its behavior for molecules in selected vibrational and (end-over-end) rotational states. Arguably the most interesting aspect of molecules compared to 
TABLE I. A selection of polar molecules suited for deceleration and trapping experiments, with their relevant properties.

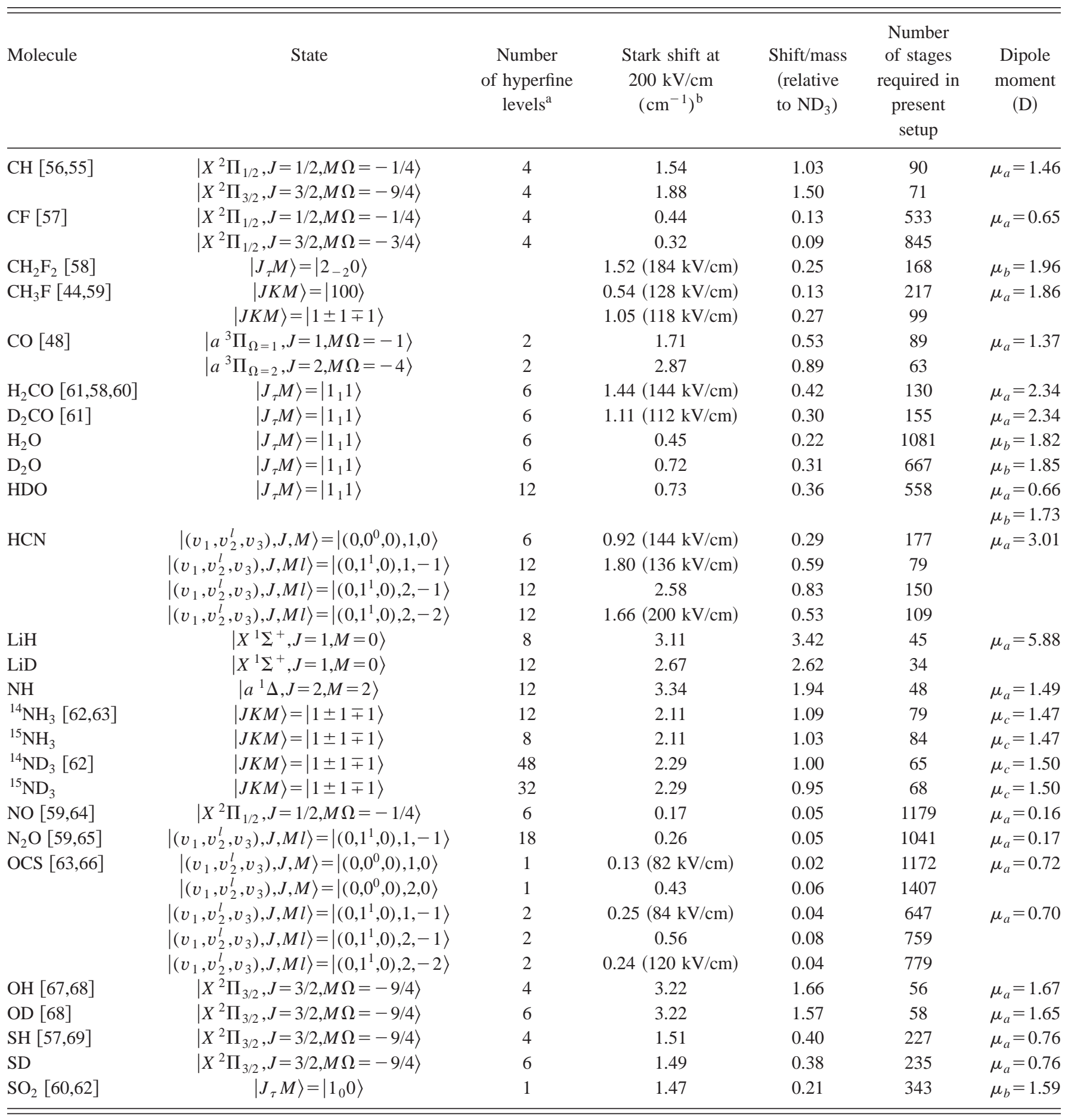

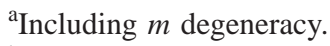

${ }^{b}$ When the Stark shift reaches a maximum at lower values of the electric field, this maximum shift is given, together with the value of the electric field (in brackets).

atoms is that many molecules have a permanent electric dipole moment. The anisotropic interaction between the dipoles will give rise to rich and new physics in the cold dipolar gases [87]. The mean-field interparticle interaction, and hence the occurrence of molecular Bose-Einstein condensation, will depend strongly on, and can therefore be manipulated by, the trapping geometry [88]. This offers new possibilities for controlling and engineering macroscopic quantum states. It has been predicted that cold dipolar gases of fermions are excellent candidates for achieving the superfluid transition [89]. At ultracold temperatures dipolar molecules are expected to form crystalline structures, similar to the formation of Wigner crystals in ion traps [90]. Another challenging prospect is that trapped polar molecules might be used for quantum computation; in this design, the qubits would be the electric dipole moments of ultracold molecules, oriented 
along or against an external electric field, with coupling between bits via the electric dipole-dipole interaction [91].

\section{ACKNOWLEDGMENTS}

This work is part of the research program of the Stichting voor Fundamenteel Onderzoek der Materie (FOM), which is financially supported by the Nederlandse Organisatie voor Wetenschappelijk Onderzoek (NWO). The research of R.T.J. has been made possible by the support from the Royal Netherlands Academy of Arts and Sciences. We acknowledge the expert technical assistance of A.J.A. van Roij. We thank P.H.M. Smeets, B. Sartakov, and G. Berden for support in the experiments and for helpful discussions.
[1] J.M. Doyle and B. Friedrich, Nature (London) 401, 749 (1999).

[2] D. Herschbach, Rev. Mod. Phys. 71, S411 (1999).

[3] C.J. Williams and P.S. Julienne, Science 287, 986 (2000).

[4] J.T. Bahns, P.L. Gould, and W.C. Stwalley, Adv. At., Mol., Opt. Phys. 42, 171 (2000).

[5] B.G. Levi, Phys. Today 53(19), 46 (2000).

[6] H.R. Thorsheim, J. Weiner, and P.S. Julienne, Phys. Rev. Lett. 58, 2420 (1987).

[7] Y.B. Band and P.S. Julienne, Phys. Rev. A 51, R4317 (1995).

[8] A.P. Mosk, M.W. Reynolds, T.W. Hijmans, and J.T.M. Walraven, Phys. Rev. Lett. 82, 307 (1999).

[9] N. Herschbach, P.J.J. Tol, W. Vassen, W. Hogervorst, G. Woestenenk, J.W. Thomsen, P. van der Straten, and A. Niehaus, Phys. Rev. Lett. 84, 1874 (2000).

[10] W.I. McAlexander, E.R.I. Abraham, N.W.M. Ritchie, C.J. Williams, H.T.C. Stoof, and R.G. Hulet, Phys. Rev. A 51, R871 (1995).

[11] P.D. Lett, K. Helmerson, W.D. Phillips, L.P. Ratliff, S.L. Rolston, and M.E. Wagshul, Phys. Rev. Lett. 71, 2200 (1993).

[12] H. Wang, P.L. Gould, and W.C. Stwalley, Phys. Rev. A 53, R1216 (1996).

[13] A.N. Nikolov, E.E. Eyler, X.T. Wang, J. Li, H. Wang, W.C. Stwalley, and P.L. Gould, Phys. Rev. Lett. 82, 703 (1999).

[14] G. Zinner, T. Binnewies, F. Riehle, and E. Tiemann, Phys. Rev. Lett. 85, 2292 (2000).

[15] J.D. Miller, R.A. Cline, and D.J. Heinzen, Phys. Rev. Lett. 71, 2204 (1993).

[16] C. Gabbanini, A. Fioretti, A. Lucchesini, S. Gozzini, and M. Mazzoni, Phys. Rev. Lett. 84, 2814 (2000).

[17] A. Fioretti, D. Comparat, A. Crubellier, O. Dulieu, F. MasnouSeeuws, and P. Pillet, Phys. Rev. Lett. 80, 4402 (1998).

[18] R. Wynar, R.S. Freeland, D.J. Han, C. Ryu, and D.J. Heinzen, Science 287, 1016 (2000).

[19] J.M. Gerton, D. Strekalov, I. Prodan, and R.G. Hulet, Nature (London) 408, 692 (2000).

[20] J.P. Shaffer, W. Chalupczak, and N.P. Bigelow, Phys. Rev. Lett. 82, 1124 (1999).

[21] U. Schlöder, C. Silber, and Z. Zimmermann, Appl. Phys. B: Lasers Opt. 73, 801 (2001).

[22] T. Takekoshi, B.M. Patterson, and R.J. Knize, Phys. Rev. Lett. 81, 5105 (1998).

[23] N. Vanhaecke, W. de Souza Melo, B. Laburthe Tolra, D. Comparat, and P. Pillet, Phys. Rev. Lett. (to be published).

[24] J.D. Weinstein, R. deCarvalho, T. Guillet, B. Friedrich, and J.M. Doyle, Nature (London) 395, 148 (1998).

[25] R. deCarvalho, J.M. Doyle, B. Friedrich, T. Guillet, J. Kim, D. Patterson, and J.D. Weinstein, Eur. Phys. J. D 7, 289 (1999).
[26] J. G. King and J. R. Zacharias, Quarterly Progress Report, Research Laboratory of Electronics, Massachusetts Institute of Technology. No. 48, 1958 (unpublished).

[27] R. Golub, Ph.D. thesis, Massachusetts Institute of Technology, 1967.

[28] D. Auerbach, E.E.A. Bromberg, and L. Wharton, J. Chem. Phys. 45, 2160 (1966).

[29] R. Wolfgang, Sci. Am. 219(4), 44 (1968).

[30] E.E.A. Bromberg, Ph.D. thesis, University of Chicago, 1972.

[31] H.L. Bethlem, G. Berden, and G. Meijer, Phys. Rev. Lett. 83, 1558 (1999).

[32] J.A. Maddi, T.P. Dinneen, and H. Gould, Phys. Rev. A 60, 3882 (1999).

[33] H.L. Bethlem, G. Berden, A.J.A. van Roij, F.M.H. Crompvoets, and G. Meijer, Phys. Rev. Lett. 84, 5744 (2000).

[34] A.J. Lichtenberg, in Phase-Space Dynamics of Particles (Wiley, New York, 1969).

[35] H.L. Bethlem, G. Berden, F.M.H. Crompvoets, R.T. Jongma, A.J.A. van Roij, and G. Meijer, Nature (London) 406, 491 (2000).

[36] F.M.H. Crompvoets, H.L. Bethlem, R.T. Jongma, and G. Meijer, Nature (London) 411, 174 (2001).

[37] V. I. Veksler, J. Phys. (USSR) 9, 153 (1945).

[38] E.M. McMillan, Phys. Rev. 68, 143 (1945).

[39] D.A. Edwards and M.J. Syphers, in An Introduction to the Physics of High Energy Accelerators (Wiley, New York, 1993).

[40] D.A. Dahl, Simion 3D Version 6.0 (Idaho National Engineering Laboratory, Idaho Falls, 1995).

[41] W.H. Wing, Prog. Quantum Electron. 8, 181 (1984).

[42] H.L. Bethlem, A.J.A. van Roij, R.T. Jongma, and G. Meijer, Phys. Rev. Lett. 88, 133003 (2002).

[43] J. van Veldhoven, R.T. Jongma, B. Sartakov, W.A. Bongers, and G. Meijer (unpublished).

[44] P.W. Harland, W.-P. Hu, C. Vallance, and P.R. Brooks, Phys. Rev. A 60, 3138 (1999).

[45] D.R. Miller, in Atomic and Molecular Beam Methods, edited by G. Scoles (Oxford University Press, New York, 1988), Vol. I.

[46] R.V. Latham, in High Voltage Vacuum Insulation: The Physical Basis (Academic Press, London, 1981).

[47] J. Reuss, in Atomic and Molecular Beam Methods (Ref. [45]).

[48] R.T. Jongma, Th. Rasing, and G. Meijer, J. Chem. Phys. 102, 1925 (1995).

[49] S.Y. Lee, in Accelerator Physics (World Scientific, Singapore, 1999).

[50] O.J. Luiten, M.W. Reynolds, and J.T.M. Walraven, Phys. Rev. A 53, 381 (1996).

[51] W.H. Wing, Phys. Rev. Lett. 45, 631 (1980). 
[52] W. Paul, Rev. Mod. Phys. 62, 531 (1990).

[53] M.N.R. Ashfold, R.N. Dixon, N. Little, R.J. Stickland, and C.M. Western, J. Chem. Phys. 89, 1754 (1988).

[54] S.Y.T. van de Meerakker, R.T. Jongma, H.L. Bethlem, and G. Meijer, Phys. Rev. A 64, 041401(R) (2001).

[55] M.A. Weibel, T.D. Hain, and T.J. Curtiss, J. Chem. Phys. 108, 3134 (1998).

[56] M. Takezaki, H. Ohoyama, T. Kasai, and K. Kuwata, Laser Chem. 15, 113 (1995).

[57] M.A. Weibel, T.D. Hain, and T.J. Curtiss, J. Vac. Sci. Technol. A 15, 2238 (1997).

[58] T.D. Hain, R.M. Moision, and T.J. Curtiss, J. Chem. Phys. 111, 6797 (1999).

[59] D.H. Parker, H. Jalink, and S. Stolte, J. Phys. Chem. 91, 5427 (1987).

[60] E.M. Jones and P.R. Brooks, J. Chem. Phys. 53, 55 (1970).

[61] N.F. van Hulst, J.J. ter Meulen, and A. Dymanus, J. Chem. Phys. 86, 1407 (1987).

[62] N.I. Butkovskaya, M.N. Larichev, I.O. Leipunskii, I.I. Morozov, and V.L. Tal'rose, Chem. Phys. 12, 267 (1976).

[63] S.R. Gandhi, Q.-X. Xu, T.J. Curtiss, and R.B. Bernstein, J. Phys. Chem. 91, 5437 (1987).

[64] J.J. van Leuken, F.H.W. van Amerom, J. Bulthuis, J.G. Snijders, and S. Stolte, J. Phys. Chem. 99, 15573 (1995).

[65] H. Jalink, F. Harren, D. van den Ende, and S. Stolte, Chem. Phys. 108, 391 (1986).

[66] S.R. Gandhi and R.B. Bernstein, J. Chem. Phys. 87, 6457 (1987).

[67] K. Schreel, J. Schleipen, A. Eppink, and J.J. ter Meulen, J. Chem. Phys. 99, 8713 (1993).

[68] T.D. Hain, M.A. Weibel, K.M. Backstrand, and T.J. Curtiss, J. Phys. Chem. A 101, 7674 (1997).

[69] T. Kasai, K. Ohashi, H. Ohoyama, and K. Kuwata, Chem. Phys. Lett. 127, 581 (1986).
[70] M.C. van Beek and J.J. ter Meulen, Chem. Phys. Lett. 337, 237 (2001).

[71] S.K. Sekatskiü, Pis'ma Zh. Eksp. Teor. Fiz. 62, 900 (1995) [JETP Lett. 62, 916 (1995)].

[72] H.J. Loesch, Chem. Phys. 207, 427 (1996).

[73] R.T. Jongma, G. von Helden, G. Berden, and G. Meijer, Chem. Phys. Lett. 270, 304 (1997).

[74] H.J. Loesch and B. Scheel, Phys. Rev. Lett. 85, 2709 (2000).

[75] D.P. Katz, J. Chem. Phys. 107, 8491 (1997).

[76] F. Shimizu and M. Morinaga, Jpn. J. Appl. Phys., Part 2 31, L1721 (1992).

[77] E. Peik, Eur. Phys. J. D 6, 179 (1999).

[78] V. Vuletić and S. Chu, Phys. Rev. Lett. 84, 3787 (2000).

[79] E.A. Hinds, Phys. Scr. T70, 34 (1997).

[80] Ch. Daussy, T. Marrel, A. Amy-Klein, C.T. Nguyen, Ch.J. Bordé, and Ch. Chardonnet. Phys. Rev. Lett. 83, 1554 (1999).

[81] J.K. Webb, M.T. Murphy, V.V. Flambaum, V.A. Dzuba, J.D. Barrow, C.W. Churchill, J.X. Prochaska, and A.M. Wolfe, Phys. Rev. Lett. 87, 091301 (2001).

[82] N. Balakrishnan, A. Dalgarno, and R.C. Forrey, J. Chem. Phys. 113, 621 (2000).

[83] W. Ketterle and N.J. van Druten, Adv. At., Mol., Opt. Phys. 37, 181 (1996)

[84] J.L. Bohn, Phys. Rev. A 63, 052714 (2001).

[85] E.A. Cornell, C. Monroe, and C.E. Wieman, Phys. Rev. Lett. 67, 2439 (1991).

[86] G. Modugno, G. Ferrari, G. Roati, R.J. Brecha, A. Simoni, and M. Inguscio, Science 294, 1320 (2001).

[87] M. Baranov, Ł. Dobrek, K. Góral, L. Santos, and M. Lewenstein, e-print cond-mat/0201100.

[88] L. Santos, G.V. Shlyapnikov, P. Zoller, and M. Lewenstein, Phys. Rev. Lett. 85, 1791 (2000).

[89] M.A. Baranov, M.S. Mar'enko, Val.S. Rychkov, and G.V. Shlyapnikov, e-print cond-mat/0109437.

[90] G.V. Shlyapnikov (private communications).

[91] D. DeMille, Phys. Rev. Lett. 88, 067901 (2002). 